\title{
Influence of the characteristics of the experimental data set used to identify anisotropy parameters
}

\author{
Ali Khalfallah ${ }^{\mathrm{a}, \mathrm{b}, *}$, José Luís Alves $^{\mathrm{c}}$, Marta Cristina Oliveira ${ }^{\mathrm{a}}$, Luís Filipe Menezes ${ }^{\mathrm{a}}$ \\ a CEMUC, Department of Mechanical Engineering, University of Coimbra, Polo II, Rua Luís Reis Santos, Pinhal de Marrocos, 3030-788 Coimbra, Portugal \\ ${ }^{\mathrm{b}}$ LGM, Department of Mechanical Engineering, Ecole Nationale d'Ingénieurs de Monastir, Monastir, Tunisia \\ ${ }^{\mathrm{C}}$ CT2M, Department of Mechanical Engineering, University of Minho, Campus de Azurém, 4800-058 Guimarães, Portugal
}

\section{A R T I C L E I N F O}

\section{Article history:}

Received 3 July 2014

Received in revised form 17 February 2015

Accepted 21 February 2015

\section{Keywords:}

Constitutive modelling

Parameter identification

Plastic anisotropy

Sheet metal forming

\begin{abstract}
A B S T R A C T
This work presents an investigation into the effect of the number and type of experimental input data used in parameter identification of Hill'48, Barlat'91 (Yld91) and Cazacu and Barlat'2001 (CB2001) yield criteria on the accuracy of the finite element simulation results. Different sets of experimental data are used to identify the anisotropy parameters of two metal sheets, exhibiting different anisotropic behaviour and hardening characteristics: a mild steel (DC06) and an aluminium alloy (AA6016-T4). Although it has been shown that the CB2001 yield criterion can lead to an accurate description of anisotropic behaviour of metallic sheets, its calibration requires a large set of experimental input data. A calibration procedure is proposed for $\mathrm{CB} 2001$ based on a reduced set of experimental data, i.e. where the results are limited to three uniaxial tensile tests, combined with artificial data obtained using the Barlat'91 yield criterion. Evaluation of the predictive capacity of the studied yield criteria, calibrated using different sets of experimental data, is made by comparing finite element simulation results with experimental results for the deep drawing of a crossshaped part. A satisfying agreement is observed between experimental and numerical thickness distributions, with a negligible effect of the number and type of experimental data for the Hill'48 and Yld91 yield criteria. On the contrary, CB2001 calibration is quite sensitive to the experimental data available, particularly biaxial values. Nevertheless, CB2001 calibration based on the combination of effective and artificial experimental data achieves satisfying results, which in the worst case are similar to the ones obtained with the Yld91.
\end{abstract}

(c) 2015 Elsevier B.V. All rights reserved.

\section{Introduction}

The automotive and aeronautical industries are continuously clamouring for new or improved materials for developing better-quality structural parts. The resulting materials are commonly characterised by complex behaviour. This demand must be accompanied by the development of more reliable constitutive models to meet accurate finite element (FE) simulation results. Nowadays, FE simulation is an essential tool for testing and developing new parts, particularly in industrial environments, owing to the associated significant reduction in expensive experimental costs. However, FE predictions are highly dependent on accurately describing the material's mechanical behaviour, i.e. the selected constitutive model as well as the input data used for its calibration. Nonetheless, accurately describing the material's behaviour also depends on the number

\footnotetext{
* Corresponding author at: LGM, Department of Mechanical Engineering, Ecole Nationale d'Ingénieurs de Monastir, Monastir, Tunisia.
} 
and type of available data measurements from mechanical tests. In fact, over recent decades several advanced constitutive models have been proposed and they require a large number of varied experimental data for their calibration. These phenomenological models are typically based on the use of plastic potentials and associated flow rules and have been successfully applied in FE analysis of deep drawing processes. Hence, they are successfully and intensively implemented in both academic and commercial FE packages. The yield criteria of Tresca and von Mises are the most widely used for describing isotropic metallic materials. The latter criterion was the basis for the development of the first orthotropic yield criterion, proposed in 1948 by Hill (Hill48) [1]. Nowadays, von Mises and Hill48 are the most implemented of all yield criteria in FE codes. Later, Hershey [2] and Hosford [3] introduced a generalised isotropic yield function with a variable exponent that takes into account the material's crystallographic structure [4]. This yield function was generalised for anisotropic metals by Barlat et al. [5]. Karafillis and Boyce [6] introduced the anisotropy parameters using the concept a fourth-order tensor in a linear transformation of the Cauchy stress tensor. The linear transformation approach, applied to the deviatoric stress tensor, is attractive since it assures the yield function's convexity and is a convenient means of introducing a large number of anisotropy parameters in the new yield criteria formulation, increasing their flexibility [7]. Based on this approach, Barlat et al. [8] proposed an extension of Yld91, the so-called Yld96, which involves seven anisotropy coefficients under a plane stress state. Later, a plane stress yield function using two linear transformations of the deviatoric stress tensor, referred to as Yld2000-2d, was proposed. This yield criterion involves eight independent anisotropy coefficients [9]. Thereafter, Barlat et al. [10] developed the Yld2004-18p yield criterion, also based on two linear transformations, which was established for a pressure independent material under general stress conditions. For plane stress conditions, the eighteen independent anisotropy parameters involved in this yield function are reduced to fourteen [11]. Furthermore, it has been shown that Yld89 [12] and Yld91 yield criteria [5] are particular cases of the more recently proposed criteria, i.e. Yld2000-2d and Yld2004-18p, respectively [7].

Aretz and Barlat [13] suggested a new yield function for orthotropic metal sheets, considered to be complementary to the Yld2004-18p and so-called Yld2011-18p. The later was extended to the so-called Yld2011-27p yield criterion, which involves a total of twenty-seven anisotropy parameters. This shows that the concept of using multiple linear transformations of the deviatoric stress tensor enables the construction of more flexible yield criteria, associated with a larger number of anisotropy coefficients. However, using multiple linear transformations, analytical computation of the yield function gradient is quite complex and its numerical implementation is prone to errors [13].

Aware of the limitations of quadratic yield criteria, Hill proposed a general homogeneous polynomial formulation as a yield function for plane stress states [14]. Gotoh was the first to explore the use of a fourth-order polynomial as a plane stress yield function [15]. Gotoh suggested the use of eight directional uniaxial tensile properties and the balanced biaxial stress to identify the nine anisotropy parameters of the yield criterion. It was the first yield function that could simultaneously describe both directional yield stresses and $r$-values. Nevertheless, the shortcoming of using a polynomial function for yield criterion formulation is the lack of evidence of convexity conditions. Additionally, any anisotropic yield function should be reduced to an isotropic function by imposing constraints on its anisotropy parameters. In this context, Cazacu and Barlat proposed an extension of Drucker's isotropic criterion [16] to anisotropy, based on the formulation of a sixth-order polynomial function. The CB2001 yield criterion involves eighteen anisotropy parameters under a general stress state and eleven coefficients for plane stress conditions, enabling the description of a wide range of anisotropic material properties [17,18].

Concurrently, Banabic et al. [19] developed a plane stress yield criterion, referred to as BBC2000, which was derived from the criterion proposed by Barlat and Lian in 1989 (Yld89) [12]. The Yld89 yield function uses eight anisotropy parameters to describe anisotropic behaviour, along with an exponent coefficient related to the material crystallographic structure [4]. Banabic et al. improved the BBC2000 criterion by rearranging the anisotropy parameters, offering more possibilities for adjusting these parameters to the experimental data. This yield criterion, referred to as BBC2005, encompasses eight independent anisotropy coefficients [20,21]. Barlat analysed Yld2000-2d, BBC2005 and the yield function proposed by Aretz in 2004 [22,23] and pointed out that for plane stress conditions, these yield criteria are similar [7]. Comsa and Banabic formulated BBC2008 as a finite series of anisotropy parameters that can be expanded or reduced from eight to twenty-four parameters, depending on the available number of experimental data measurements [24]. Vrh et al. demonstrated the ability of the BBC2008 yield criterion to predict the earing profiles in a cylindrical deep drawing cup, for two anisotropic aluminium alloys [25].

Based on this brief literature review, it can be noted that several sophisticated yield criteria are being continuously proposed, trying to enhance the description of the anisotropic plastic behaviour of metal sheets. Moreover, it has been shown that the ability of a yield criterion to describe the material's anisotropic behaviour depends on its flexibility, which in turn is related to the number of anisotropic parameters. As a consequence, the necessary number and type of experimental tests for the calibration of flexible yield criteria increases $[13,25,26]$. However, the cost associated with a large number of different types of experimental tests along with the mathematical complexity of the advanced yield criteria are the major shortcomings contributing to a limited use in an industrial environment. In order to enhance the application of advanced yield criteria in the sheet metal forming industry, some attempts have been made to narrow the gap between yield function calibration costs and its efficiency for describing anisotropic material behaviour. In this sense, it is of paramount importance to investigate less expensive parameter identification approaches, with the aim of reducing the required sets of experimental input data while maintaining reasonable accuracy. Therefore, Malo et al. [27] proposed a strategy to determine the anisotropy parameters of the Hill48 and Yld89 yield criteria using stress data obtained from tensile and bending tests. However, no validation tests were performed to assess the efficiency of the identification strategy. Wu et al. [28] investigated the 
predictive capacity of Barlat et al. (Yld91 [5], Yld96 [8]) and the Karafillis and Boyce (KB93 [6]) yield criteria based on a comparison of predicted and experimental yield stresses and $r$-values. Forming limit diagrams (FLD) were also produced to assess yield function competence based on the comparison between predicted and experimental curves. It has been shown that KB93 and Yld96 perform better than Yld91, since the predictive capacity of the latter depends on whether the anisotropy parameters were calculated from the yield stresses or the $r$-values.

According to Barlat [7], Banabic and Comsa [21], the balance between accuracy and parameter identification costs (i.e. efficiency for practical applications) of advanced yield criteria is roughly attained by using a set of seven or eight experimental values. Taking into account sensitivity analysis results, six input values are commonly used for parameter identification: the three yield stresses and three $r$-values obtained from three directional uniaxial tensile tests, performed using orientations of $0^{\circ}, 45^{\circ}$ and $90^{\circ}$ to the rolling direction. However, six experimental values are insufficient to calibrate recent advanced yield criteria which include at least seven anisotropy parameters. Thus, additional experimental values are needed, such as the balanced biaxial stress $\sigma_{b}$, measured from either the bulge test [29] or the equibiaxial tensile test [20], as well as the balanced biaxial strain ratio $r_{b}$-value, obtained from either the disk compression test [9] or the equibiaxial tensile test. Many researchers recommend the use of balanced biaxial data, whenever possible [7,20], to improve the adjustment of the yield surface for the biaxial loading mode. However, Banabic et al. carried out a sensitivity analysis on the influence of the balanced biaxial stress $\sigma_{b}$ and the equibiaxial strain ratio ( $r_{b}$-value) based on the FE results of a cross-die test. The results showed that the balanced biaxial stress $\sigma_{b}$ has a higher effect on predicted results than the $r_{b}$-value. Hence, Banabic reported that measuring the $r_{b}$-value is not recommend for practical use with the BBC2005 yield criterion [21]. Contrarily, Aretz [30] showed that the $r_{b}$-value has a substantial impact on the yield locus's shape and, as a consequence, on the predicted forming limit diagrams (FLD).

Paraianu et al. [31] identified BBC2005 anisotropy parameters using different sets of experimental data to study the influence of the number and type of experimental data used on the accurate prediction of the forming limit curves (FLD). They have shown that the use of seven or eight input values leads to a good level of agreement between predicted and experimental results. Lazarescu et al. [26] investigated the influence of the input data used to calibrate yield criteria parameters on the accurate prediction of hydraulic bulge test results. The predictions obtained with BBC2005, Hill48 and Yld89 yield criteria are compared to experimental ones, showing that BBC2005 leads to the best agreement. Lazarescu et al. [26] provides a rough guide, stating that the Yld89 criterion is better at predicting the biaxial yield stress than Hill48, which underestimates the experimental data for the AA6016-T4 aluminium alloy. As an alternative to the extensively used conventional calibration procedures which are based on uniaxial tensile and equibiaxial tests, Aretz put forward a calibration method involving three directional uniaxial tensile tests and two plane strain tensile tests, performed in the rolling and transverse directions. This method was applied to the Yld2003 [22], a non-quadratic plane stress yield criterion [32], and its performance was assessed based on the comparison of the predicted FLD curve with that obtained by using the conventional calibration procedure. It was shown that the proposed calibration method gives satisfactory results. However, the success of such a procedure depends strongly on the accuracy of the experimental plane strain tensile tests, which is greatly dependent on the sample geometry.

The present study investigates the influence of the characteristics of the experimental data set (used in the parameter identification of anisotropic yield criterion) on FE sheet metal forming process results. The Hill, 1948 (Hill48) [1], Barlat, 1991 (Yld91) [5] and Cazacu and Barlat, 2001 (CB2001) [33] yield criteria are considered. CB2001 is known as a flexible yield criterion, suitable for predicting the behaviour of highly anisotropic materials. However, the main drawback for its industrial application is the large set of experimental input data required to identify the eleven anisotropy parameters in the case of plane stress state. Thus, an alternative calibration strategy is proposed for CB2001, using a limited set of experimental data, trying to highlight its advantages when compared with the other yield criteria studied.

\section{Constitutive equations}

Consider a material point belonging to a continuum medium with elastoplastic behaviour and subjected to a given loading. The total deformation gradient $\underline{F}=\partial \underline{x} / \partial \underline{X}$ can be decomposed into elastic and plastic parts, $\underline{F}=\underline{F}^{\mathrm{el}} \underline{F}^{\mathrm{pl}}$, where $\underline{F}^{\mathrm{el}}$ and $\underline{F}^{\mathrm{pl}}$ are the elastic and plastic parts, respectively. The velocity gradient $\underline{L}$ can also be decomposed into its elastic and plastic parts, $\underline{L}=\underline{L}^{\mathrm{el}}+\underline{L}^{\mathrm{pl}}$, which further allows decomposition of the total strain-rate tensor $\underline{\dot{\varepsilon}}$ into elastic $\left(\underline{\dot{\varepsilon}}^{\mathrm{el}}\right)$ and plastic $\left(\underline{\dot{\varepsilon}}^{\mathrm{pl}}\right)$ parts,

$$
\underline{\dot{\varepsilon}}=\underline{\dot{\varepsilon}}^{\mathrm{el}}+\underline{\dot{\varepsilon}}^{\mathrm{pl}} \text {. }
$$

The elastic and plastic parts of the strain-rate tensor are additive. Besides this, metallic materials usually have much smaller elastic strains than plastic strains.

The generalised form of Hooke's law can be applied to relate the Cauchy stress rate tensor $\underline{\dot{\sigma}}$ with the elastic strain-rate tensor $\underline{\dot{\varepsilon}}^{\text {el }}$ :

$$
\underline{\dot{\sigma}}=\underline{\underline{C}}: \underline{\dot{e}}^{\mathrm{el}}
$$

where $\underline{\underline{C}}$ is a fourth-order tensor which denotes the second-order elastic constant's tensor. Assuming that the elastic behaviour is isotropic, $\underline{\underline{C}}$ can be given in terms of the Young modulus $E$ and the Poisson ratio $v$ as: 


$$
\underline{\dot{\sigma}}=\frac{E}{(1+v)}\left(\underline{\dot{\varepsilon}}^{\mathrm{el}}+\frac{v}{1-2 v} \operatorname{tr}\left(\underline{\dot{\varepsilon}}^{\mathrm{el}}\right) \underline{I}\right)
$$

where $I$ is the second-order identity tensor.

To describe the plastic behaviour, it is necessary to define: (i) a plastic potential; (ii) a flow rule and (iii) a hardening law. The yield criterion plays the role of a plastic potential and the hardening law defines the evolution of the yield surface with respect to the plastic work. The yielding is defined through function $f$, given by:

$$
f(\bar{\sigma}, Y)=\bar{\sigma}-Y=0
$$

where $\bar{\sigma}$ is the equivalent stress, given by the yield criterion, and $Y$ is the flow stress, whose evolution is given by the isotropic hardening law. Thus, $Y$ is a scalar measurement of the size of the yield surface (strength of the material).

For metallic materials an associated flow rule is usually adopted. This means that the plastic strain-rate tensor is coupled with the stress state. Therefore, the plastic strain-rate tensor is derived from the yield function, defined in the stress space, as follows:

$$
\underline{\dot{\varepsilon}}^{\mathrm{pl}}=\dot{\lambda} \frac{\partial f}{\partial \underline{\sigma}}
$$

where $\underline{\dot{\varepsilon}}^{\text {pl }}$ is the plastic strain-rate tensor and $\underline{\sigma}$ represents the Cauchy stress tensor, both defined in the orthotropic material's frame. The so-called plastic multiplier is given by $\lambda$. If the equivalent stress $(\bar{\sigma})$ is a first-order homogeneous function of stresses, then through the work-equivalence principle it follows that the evolution law of the equivalent plastic strain-rate $\dot{\bar{\varepsilon}}^{\text {pl }}$ (conjugated with $\bar{\sigma}$ ) reduces to $\dot{\lambda}=\dot{\bar{\varepsilon}}^{\text {pl }}$ and then, under a plastic loading,

$$
\dot{\bar{\varepsilon}}^{\mathrm{pl}}>0 ; \quad \dot{\bar{\varepsilon}}^{\mathrm{pl}} f=0 ; \quad \dot{\bar{\varepsilon}}^{\mathrm{pl}} \dot{f}=0 .
$$

\subsection{Hill, 1948 (Hill48)}

In order to describe the anisotropic behaviour of metallic materials, Hill proposed a generalisation of von Mises's distortional energy quadratic criterion [1]. This criterion is used to describe the orthotropic behaviour of conventional steels, such as rolled steel, since it enables an accurate description of these materials, particularly in the case of low textured steels. In addition, due to its simple quadratic form, it is easily implemented in FE codes. The 3D form of the Hill48 yield criterion is expressed by:

$$
H\left(\sigma_{11}-\sigma_{22}\right)^{2}+F\left(\sigma_{22}-\sigma_{33}\right)^{2}+G\left(\sigma_{33}-\sigma_{11}\right)^{2}+2 N \tau_{12}^{2}+2 L \tau_{23}^{2}+2 M \tau_{31}^{2}=\bar{\sigma}^{2}
$$

For metal sheets, the $L$ and $M$ parameters cannot be evaluated, thus the corresponding isotropy condition values are commonly adopted, i.e. 1.5. Therefore, the anisotropy parameters reduce to $F, G, H$, and $N$.

\subsection{Barlat, 1991 (Yld91)}

Barlat proposed a non-quadratic yield criterion based on a linear transformation of the stress tensor [5] for orthotropic materials, given by:

$$
\left|s_{1}-s_{2}\right|^{m}+\left|s_{1}-s_{3}\right|^{m}+\left|s_{2}-s_{3}\right|^{m}=2 \bar{\sigma}^{m}
$$

where $s_{1}, s_{2}$ and $s_{3}$ are the principal values of a so-called isotropic equivalent stress tensor $\underline{s}$, obtained from a linear transformation $\underline{\underline{L}}$ (a fourth-order tensor), which multiplicatively operates on the Cauchy deviatoric stress tensor $\underline{\sigma}$ acting on the material,

$$
\underline{s}=\underline{\underline{L}}: \underline{\sigma} .
$$

Taking into account its major and minor symmetries, the fourth-order linear transformation operator $\underline{\underline{L}}$ can be represented by a $6 \times 6$ matrix (Voigt notation) in the orthotropic axis of symmetry (rolling direction (RD), transverse direction (TD) and normal direction (ND)), defined as follows:

$$
\underline{L}=\left[\begin{array}{cccccc}
\frac{c_{2}+c_{3}}{3} & -\frac{c_{3}}{3} & -\frac{c_{2}}{3} & 0 & 0 & 0 \\
-\frac{c_{3}}{3} & \frac{c_{3}+c_{1}}{3} & -\frac{c_{1}}{3} & 0 & 0 & 0 \\
-\frac{c_{2}}{3} & -\frac{c_{1}}{3} & \frac{c_{1}+c_{2}}{3} & 0 & 0 & 0 \\
0 & 0 & 0 & c_{4} & 0 & 0 \\
0 & 0 & 0 & 0 & c_{5} & 0 \\
0 & 0 & 0 & 0 & 0 & c_{6}
\end{array}\right]
$$


The anisotropy parameters of this yield criterion are $c_{k}(k=1, \ldots, 6)$. The shape of the yield surface changes isotropically when $m$ changes, and thus $m$ cannot really be considered to be an additional anisotropy parameter. From Logan and Hosford [4], $m$ can be considered as being equal to 6 and 8, for BCC and FCC materials, respectively. For metal sheets, parameters $c_{4}$ and $c_{5}$ cannot be evaluated, thus the corresponding isotropy condition values are commonly adopted, i.e. 1.0. Therefore, the anisotropy parameters reduce to $c_{1}, c_{2}, c_{3}$ and $c_{6}$.

\subsection{Cazacu and Barlat, 2001 (CB2001)}

The yield function of Cazacu and Barlat [33] is a generalisation of Drucker's isotropic yield criterion [16] for orthotropic materials. The CB2001 criterion is based on a generalisation of the second $J_{2}^{0}$ and the third $J_{3}^{0}$ invariants of the deviatoric Cauchy stress tensor, which are used to extend Drucker's isotropic yield criterion to orthotropy, as follows:

$$
\left(J_{2}^{0}\right)^{3}-c\left(J_{3}^{0}\right)^{2}=27\left(\frac{\bar{\sigma}}{3}\right)^{6}
$$

The second and third generalised invariants of the effective stress tensor are given by:

$$
\begin{aligned}
J_{2}^{0}= & \frac{a_{1}}{6}\left(\sigma_{11}-\sigma_{22}\right)^{2}+\frac{a_{2}}{6}\left(\sigma_{22}-\sigma_{33}\right)^{2}+\frac{a_{3}}{6}\left(\sigma_{11}-\sigma_{33}\right)^{2}+a_{4} \sigma_{12}^{2}+a_{5} \sigma_{13}^{2}+a_{6} \sigma_{23}^{2} \\
J_{3}^{0}= & \frac{1}{27}\left(b_{1}+b_{2}\right) \sigma_{11}^{3}+\frac{1}{27}\left(b_{3}+b_{4}\right) \sigma_{22}^{3}+\frac{1}{27}\left(2\left(b_{1}+b_{4}\right)-b_{2}-b_{3}\right) \sigma_{33}^{3}-\frac{1}{9}\left(b_{1} \sigma_{22}+b_{2} \sigma_{33}\right) \sigma_{11}^{2}-\frac{1}{9}\left(b_{3} \sigma_{33}+b_{4} \sigma_{11}\right) \sigma_{22}^{2} \\
& -\frac{1}{9}\left(\left(b_{1}-b_{2}+b_{4}\right) \sigma_{11}+\left(b_{1}-b_{3}+b_{4}\right) \sigma_{22}\right) \sigma_{33}^{2}+\frac{2}{9}\left(b_{1}+b_{4}\right) \sigma_{11} \sigma_{22} \sigma_{33}-\frac{1}{3}\left(2 b_{9} \sigma_{22}-b_{8} \sigma_{33}-\left(2 b_{9}-b_{8}\right) \sigma_{11}\right) \sigma_{13}^{2} \\
& -\frac{1}{3}\left(2 b_{10} \sigma_{33}-b_{5} \sigma_{22}-\left(2 b_{10}-b_{5}\right) \sigma_{11}\right) \sigma_{12}^{2}-\frac{1}{3}\left(\left(b_{6}+b_{7}\right) \sigma_{11}-b_{6} \sigma_{22}-b_{7} \sigma_{33}\right) \sigma_{23}^{2}+2 b_{11} \sigma_{12} \sigma_{23} \sigma_{13}
\end{aligned}
$$

where $a_{k}(k=1, \ldots, 6), b_{k}(k=1, \ldots, 11)$ and $c$ are the anisotropy parameters of the CB2001 yield criterion. For isotropic conditions $a_{k}$ and $b_{k}$ reduce to unity and $-3.375 \leqslant c \leqslant 2.25$. However, the conditions that guarantee the convexity of CB2001 are unknown. Therefore, following Soare et al. [17], the minimisation process adopted includes testing the convexity of the yield surface for several planes in the stress space [34]. For metal sheets the parameters $a_{5}, a_{6}$ and $b_{k}(k=6,7,8,9,11)$ cannot be evaluated, thus the corresponding isotropy condition values are commonly adopted, i.e. 1.0. Therefore, the anisotropy parameters reduce to 11 , which are: $a_{k}(k=1, \ldots, 4), b_{k}(k=1,2,3,4,5,10)$ and $c$.

\subsection{Hardening laws}

The hardening law describes the evolution of the yield surface during plastic deformation, which can be of two types: isotropic and kinematic. Isotropic hardening laws describe the change in size of the yield surface, without affecting its shape. Kinematic hardening laws express the translational motion of the yield surface centre and are therefore recommended for describing plastic deformation under strain path changes - mainly strain path reversal, in materials that exhibit Bauschinger effect. The combination of isotropic and kinematic hardening laws provides a flexible model, simultaneously describing the change in size and position of the centre of the yield surface during plastic deformation. The identification of the parameters of the isotropic hardening law requires monotonous experimental tests to be conducted. Identifying the kinematic hardening law parameters necessitates the use of cyclic tests involving strain path reversal.

Assuming that metal sheets present isotropic hardening, only one scalar parameter is required to describe the evolution of the yield surface. This scalar parameter is the equivalent plastic strain, defined as the time integral of the equivalent plastic strain-rate.

$$
\bar{\varepsilon}^{p l}=\int_{0}^{T} \dot{\bar{\varepsilon}}^{p l} d t
$$

The most used isotropic hardening laws are those of Swift and Voce. Swift's law is defined by the following equation [35]:

$$
Y\left(\bar{\varepsilon}^{\mathrm{pl}}\right)=K\left(\varepsilon_{0}+\bar{\varepsilon}^{\mathrm{pl}}\right)^{n}
$$

where $K, \varepsilon_{0}$ and $n$ are constitutive parameters. The Swift hardening law is typically considered suitable to describe the flow stress of steels. For materials exhibiting saturation of the flow stress with plastic work, as is generally the case with aluminium alloys, Voce's isotropic hardening law is the most appropriate [36]. This law is defined by the following expression:

Table 1

Isotropic hardening parameters: Swift's law for DC06.

\begin{tabular}{llll}
\hline DC06 & $Y_{0}(\mathrm{MPa})$ & $K(\mathrm{MPa})$ & $n$ \\
\hline & 123.0 & 536.94 & 0.282 \\
\hline
\end{tabular}


Table 2

Isotropic hardening parameters: Voce's law for AA6016-T4.

\begin{tabular}{llll}
\hline AA6016-T4 & $Y_{0}(\mathrm{MPa})$ & $Y_{\text {sat }}(\mathrm{MPa})$ & $C_{y}$ \\
\hline & 125.51 & 330.27 & 9.078 \\
\hline
\end{tabular}

Table 3

Experimental data for DC06 mild steel with initial thickness of $0.8 \mathrm{~mm}$ [37].

\begin{tabular}{clllllll}
\hline $\begin{array}{l}\text { Angle from } \\
\mathrm{RD}\left({ }^{\circ}\right)\end{array}$ & $\begin{array}{l}r \text {-value } \\
(-)\end{array}$ & $\begin{array}{l}\text { Uniaxial stress at } \\
\bar{\varepsilon}^{\mathrm{pl}}=10 \%\end{array}$ & $\begin{array}{l}\text { Shear stress at } \\
\bar{\varepsilon}^{\mathrm{pl}}=10 \%(\mathrm{MPa})\end{array}$ & $\begin{array}{l}\text { Biaxial stress } \\
(\mathrm{MPa})\end{array}$ & $\begin{array}{l}\text { Biaxial } r- \\
\text { value }(-)\end{array}$ & $\begin{array}{l}\text { Normal anisotropy } \\
\text { Coefficient } \bar{r}\end{array}$ & $\begin{array}{l}\text { Planar anisotropy } \\
\text { coefficient } \Delta r\end{array}$ \\
\hline 0 & 2.53 & 292.8 & 136.3 & 142.2 & 0.90 & 2.23 & 0.79 \\
15 & 2.05 & 294.5 & - & & & & \\
30 & 1.87 & 298.5 & 138.0 & & & & \\
45 & 1.84 & 303.5 & 136.5 & & & \\
60 & 2.22 & 297.8 & 140.3 & & & \\
75 & 2.62 & 297.9 & - & & & \\
90 & 2.72 & 287.5 & 138.5 & & & & \\
\hline
\end{tabular}

Table 4

Experimental data for AA6016-T4 aluminium alloy with initial thickness of $1.0 \mathrm{~mm}$ [37].

\begin{tabular}{clllllll}
\hline $\begin{array}{l}\text { Angle from } \\
\operatorname{RD}\left({ }^{\circ}\right)\end{array}$ & $\begin{array}{l}r \text { - } \\
\text { value }\end{array}$ & $\begin{array}{l}\text { Uniaxial Stress }(\mathrm{MPa}) \\
\text { at } \bar{\varepsilon}^{\mathrm{pl}}=10 \%\end{array}$ & $\begin{array}{l}\text { Shear stress }(\mathrm{MPa}) \text { at } \\
\bar{\varepsilon}^{\mathrm{pl}}=10 \%\end{array}$ & $\begin{array}{l}\text { Biaxial stress } \\
(\mathrm{MPa})\end{array}$ & $\begin{array}{l}\text { Biaxial } r- \\
\text { value }(-)\end{array}$ & $\begin{array}{l}\text { Normal anisotropy } \\
\text { Coefficient } \bar{r}\end{array}$ & $\begin{array}{l}\text { Planar anisotropy } \\
\text { coefficient } \Delta r\end{array}$ \\
\hline 0 & 0.71 & 253.9 & 137.0 & 123.4 & 1.10 & 0.63 & 0.13 \\
15 & 0.65 & 263.3 & - & & & \\
30 & 0.62 & 260.0 & 130.3 & & & \\
45 & 0.57 & 255.2 & 136.3 & & & \\
60 & 0.62 & 256.9 & 132.0 & & & \\
75 & 0.67 & 260.6 & - & & & \\
90 & 0.69 & 257.3 & 134.3 & & & & \\
\hline
\end{tabular}

$$
Y\left(\bar{\varepsilon}^{\mathrm{pl}}\right)=Y_{0}+\left(Y_{\mathrm{sat}}-Y_{0}\right)\left(1-\exp \left(-C_{\mathrm{y}} \bar{\varepsilon}^{\mathrm{pl}}\right)\right)
$$

where $Y_{0}$ is the initial yield stress, $Y_{\text {sat }}$ is the saturated flow stress and $C_{\mathrm{y}}$ is the rate of saturation.

\section{Constitutive parameter identification}

\subsection{Available experimental data}

The experimental database used in this work considers a set of tests performed for two metal sheets commonly used in the automotive industry [37]. DC06 is a mild steel (0.8 mm thick) and it has a strong anisotropic behaviour. AA6016-T4 is an aluminium alloy ( $1.0 \mathrm{~mm}$ thick) that has a reasonable combination of ductility and mechanical strength and its main application is the manufacturing of external automotive components. DC06 and AA6016-T4 have BCC and FCC crystallographic structure, respectively. The experimental database comprises $r$-values and tensile yield stresses for seven directions to $\mathrm{RD}$, shear yield stresses obtained from monotonic shear tests performed at five angles to RD and the balanced biaxial values (i.e. biaxial stress and biaxial $r$-value).

Since the experimental database comprises only monotonic experimental tests, kinematic hardening is disregarded. In fact, this work is focused on the identification of anisotropy parameters from monotonic loading tests. Therefore, to simplify analysis of the results, the isotropic hardening law parameters were identified based on the uniaxial tensile stress-strain curve along the rolling direction, using the least-squares fitting method. However, no additional constraints are added to the set of anisotropy parameters, whatever the yield criterion adopted. Thus, the same hardening law is used to identify the different yield criteria parameters, which is not necessarily the one predicted by each yield criteria for the RD. Swift and Voce laws are adopted to describe the work hardening of the mild steel DC06 and the aluminium alloy AA6016-T4, respectively. Tables 1 and 2 present the hardening law parameters obtained for both materials.

Since the experimental determination of initial yield stresses is prone to errors, in order to reduce inaccuracies in the parameter identification procedure, the values of tensile and shear stresses used correspond to an equivalent plastic strain of 10\%. Tables 3 and 4 list the full set of experimental data available for DC06 and AA6016-T4, respectively. The planar anisotropy coefficient of DC06 is higher than that of AA6016-T4, indicating a strong anisotropic behaviour of the mild steel in 
Table 5

Anisotropy parameters for DC06 obtained by the three strategies.

\begin{tabular}{|c|c|c|c|c|c|c|}
\hline Hill48 & $F$ & $G$ & $H$ & $N$ & & \\
\hline Hill_1 & 0.3294 & 0.3830 & 0.8710 & 1.7139 & & \\
\hline Hill_2 & 0.3168 & 0.3676 & 0.8445 & 1.5862 & & \\
\hline Hill_3 & 0.2697 & 0.3013 & 0.7350 & 1.3484 & & \\
\hline Yld91 & $c_{1}$ & $c_{2}$ & $c_{3}$ & $c_{6}$ & $m$ & \\
\hline Yld91_1 & 0.8446 & 0.8853 & 1.2459 & 1.0294 & 6 & \\
\hline Yld91_2 & 0.8236 & 0.8630 & 1.2167 & 0.9936 & 6 & \\
\hline Yld91_3 & 0.7917 & 0.8193 & 1.1754 & 0.9510 & 6 & \\
\hline \multicolumn{7}{|l|}{ CB2001 } \\
\hline \multirow[t]{4}{*}{ CB2001_1 } & $a_{1}$ & $a_{2}$ & $a_{3}$ & $a_{4}$ & $c$ & \\
\hline & 1.5958 & 0.8354 & 0.8979 & 1.1941 & 1.7205 & \\
\hline & $b_{1}$ & $b_{2}$ & $b_{3}$ & $b_{4}$ & $b_{5}$ & $b_{10}$ \\
\hline & 1.5947 & 1.5598 & 1.5668 & 0.9183 & 0.5363 & 1.1341 \\
\hline \multirow[t]{4}{*}{ CB2001_2 } & $a_{1}$ & $a_{2}$ & $a_{3}$ & $a_{4}$ & c & \\
\hline & 1.4688 & 0.8113 & 0.8759 & 1.0700 & 1.5490 & \\
\hline & $b_{1}$ & $b_{2}$ & $b_{3}$ & $b_{4}$ & $b_{5}$ & $b_{10}$ \\
\hline & 1.7103 & 1.6317 & 1.6246 & 0.7716 & 0.9001 & 1.1795 \\
\hline \multirow[t]{4}{*}{ CB2001_3 } & $a_{1}$ & $a_{2}$ & $a_{3}$ & $a_{4}$ & c & \\
\hline & 1.3031 & 0.9686 & 0.9822 & 1.0471 & 1.3082 & \\
\hline & $b_{1}$ & $b_{2}$ & $b_{3}$ & $b_{4}$ & $b_{5}$ & $b_{10}$ \\
\hline & 1.5313 & 2.2471 & 2.3992 & 0.6070 & 1.6540 & 1.2480 \\
\hline
\end{tabular}

terms of the in-plane $r$-values distribution. Thus, these materials provide some diversity in terms of anisotropic behaviour, enabling an improved understanding of the influence of the number and type of experimental data used in the identification strategy on the FE results.

\subsection{Anisotropy parameter identification strategies}

The increasing complexity of the yield criteria is rewarded by enhanced accuracy of sheet metal forming simulation results [38]. Conversely, the cost associated with the parameter identification procedure increases dramatically, since it becomes computationally more time consuming and requires a large number of costly mechanical tests [13,39,40]. Conventional calibration procedures rely on results from several mechanical tests to characterise the anisotropic behaviour of metal sheets. Each test allows the determination of one point of the yield surface defined in the stress space and, for some cases, the normal to the yield surface at the same point.

The directional yield stresses, the $r$-values and the biaxial values are all a function of the anisotropy parameters. The number of anisotropy parameters is typically less than the number of experimental values, which results in an over-constrained problem. Thus, in conventional calibration procedures, the parameter identification problem is formulated as a non-linear optimisation problem. The anisotropy parameters are the solution of a minimisation problem stated using a cost function which determines the difference between the experimental results and the values calculated by the model. The cost function commonly adopted can be written as follows:

$$
\begin{aligned}
\operatorname{Cost} \text { function }(p)= & \sum_{i} w_{i}\left(\frac{\sigma_{\alpha}^{\mathrm{cal}}(p)}{\sigma_{\alpha}^{\exp }}-1\right)^{2}+\sum_{i} w_{i}\left(\frac{r_{\alpha}^{\mathrm{cal}}(p)}{r_{\alpha}^{\exp }}-1\right)^{2}+\sum_{i} w_{i}\left(\frac{\tau_{\alpha}^{\mathrm{cal}}(p)}{\tau_{\alpha}^{\exp }}-1\right)^{2}+w_{i}\left(\frac{r_{b}^{\mathrm{cal}}(p)}{r_{b}^{\exp }}-1\right)^{2} \\
& +w_{i}\left(\frac{\sigma_{b}^{\mathrm{cal}}(p)}{\sigma_{b}^{\exp }}-1\right)^{2}
\end{aligned}
$$

where $p$ is the set of material parameters to be identified for a given constitutive model. $\sigma_{\alpha}^{\exp }$ and $r_{\alpha}^{\exp }$ are the experimental yield stresses and $r$-values, obtained from uniaxial tensile tests in a particular orientation $\alpha$, with respect to RD. $\sigma_{b}^{\exp }$ and $r_{b}^{\exp }$ are the experimental biaxial test values. $\tau_{\alpha}^{\exp }$ are the experimental shear stresses, obtained from shear tests oriented at a specific angle $\alpha$ to RD. $\sigma_{\alpha}^{\mathrm{cal}}, r_{\alpha}^{\mathrm{cal}}, \sigma_{b}^{\mathrm{cal}}, r_{b}^{\mathrm{cal}}$ and $\tau_{\alpha}^{\mathrm{cal}}$ are the corresponding predictions, according to the yield criterion selected. $w_{i}$ are weighting factors that can be independently defined for each experimental value or for each group of experimental input data $[19,41]$.

In this work, the cost function is used to identify the anisotropy parameters of three yield criteria: Hill48, Yld91 and CB2001. The influence of the number and type of experimental input data on the accuracy of the results is studied using 

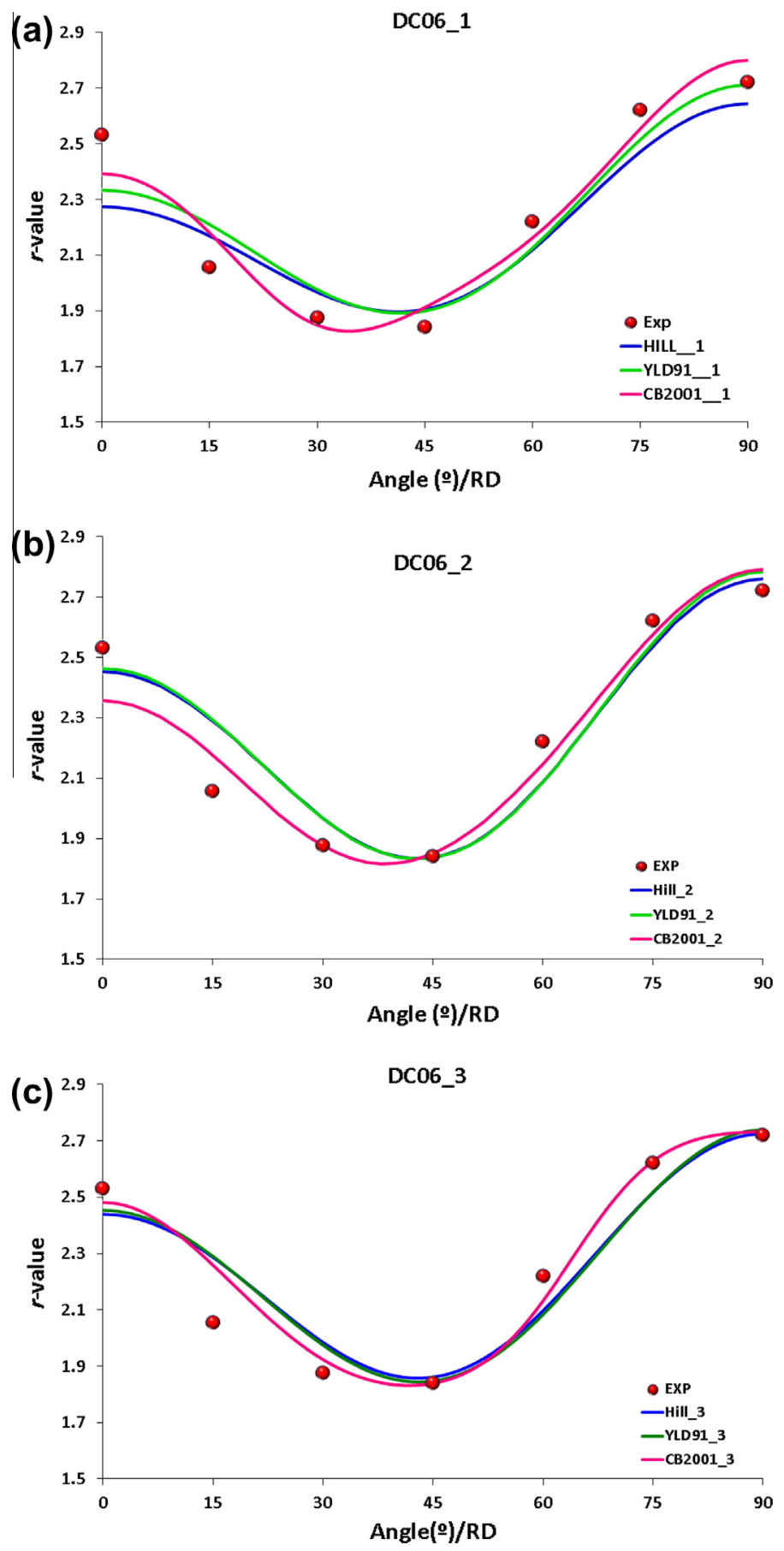

Fig. 1. Predicted $r$-values along with experimental data points for DC06 using the: (a) first strategy; (b) second strategy; and (c) third strategy.

three different sets of experimental input data. The first strategy uses all the available experimental data (i.e. 21 experimental values). In the second calibration strategy, 5 directional shear yield stresses are removed from the experimental data set. Finally, the third identification strategy considers only the results of uniaxial tensile tests. It is worth noting that the minimisation procedure is mathematically over-constrained for all identification strategies, i.e. the number of input data is larger than the number of unknown parameters, whatever the yield criteria adopted. Besides, since the aim is to evaluate the effect of the number and type of experimental data used, the cost function weighting factors are kept constant and equal to one. The use of Eq. (17) assures that the yield criteria parameters are computed in such a way that the constitutive equations associated with the yield surface reproduce - as well as possible - the experimental values. However, the Hill48 and the 

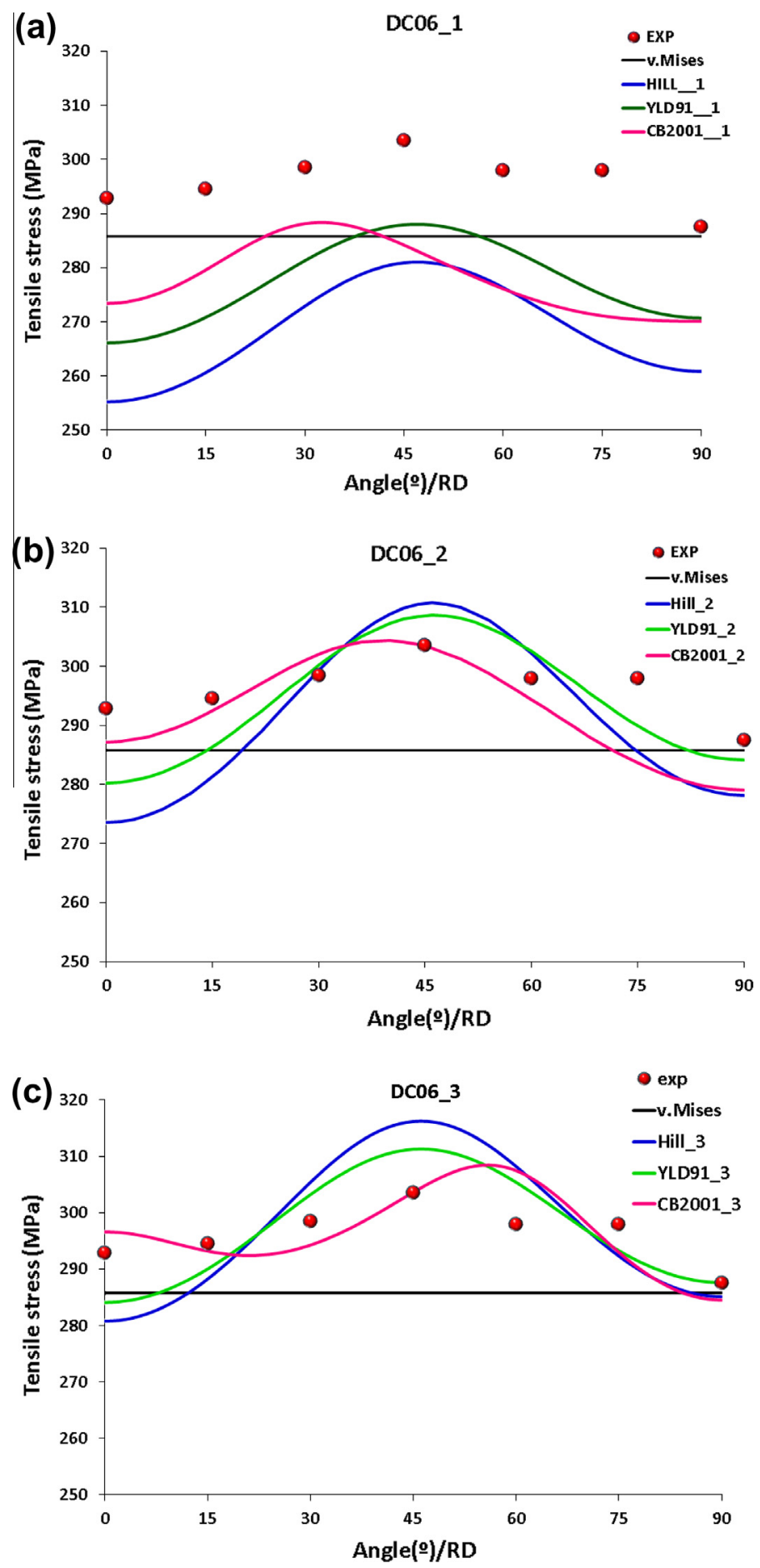

Fig. 2. Predicted tensile stresses along with experimental data points for DC06 using the: (a) first strategy; (b) second strategy; and (c) third strategy.

Yld91 cannot simultaneously describe both directional yield stresses and $r$-values. In this context, it is important to mention that the use of a weighting factor equal for both the directional yield stresses and the $r$-values leads to solutions that tend to fit the $r$-values more accurately.

All identification strategies were tested using DD3MAT, an in-house code specifically developed to identify yield criteria parameters. The algorithm implemented in DD3MAT is based on a downhill simplex method to minimise the cost function, which is a derivative free method [42]. In order to avoid falling into a local minimum, the identification procedure is 

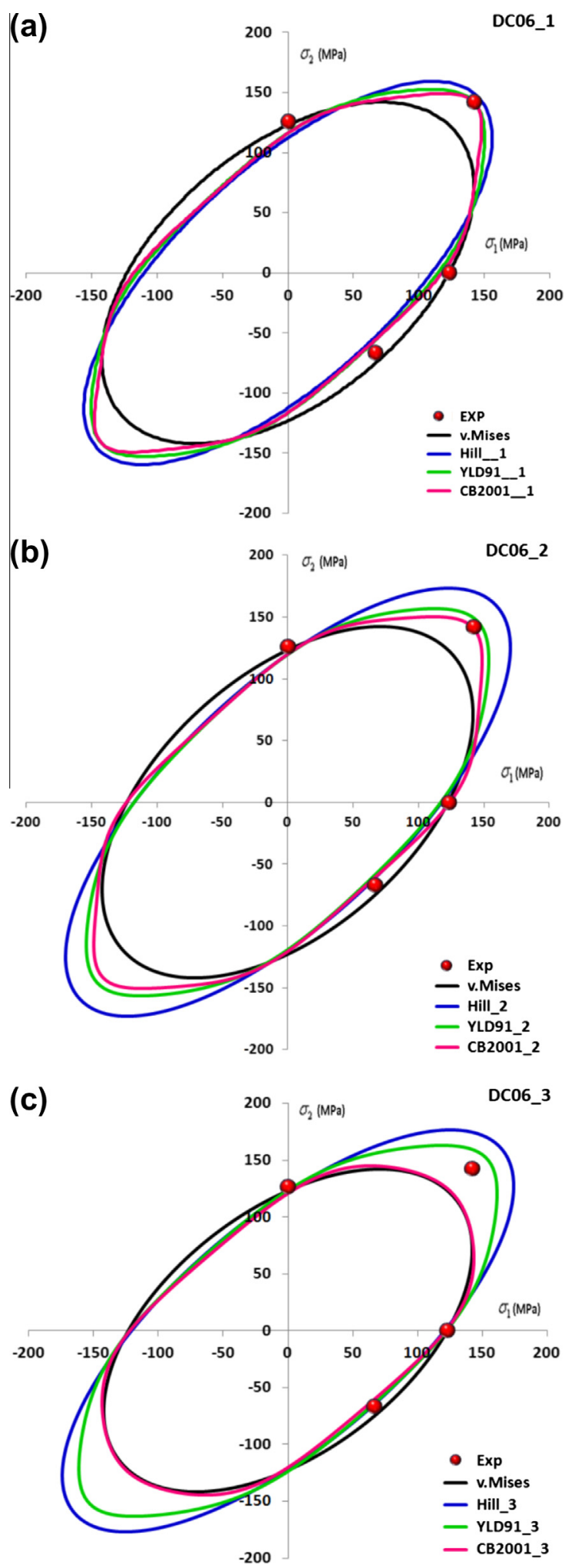

Fig. 3. Initial yield surfaces in the $\sigma_{1}$ vs. $\sigma_{2}$ space for DC06 using the: (a) first strategy; (b) second strategy; and (c) third strategy. 


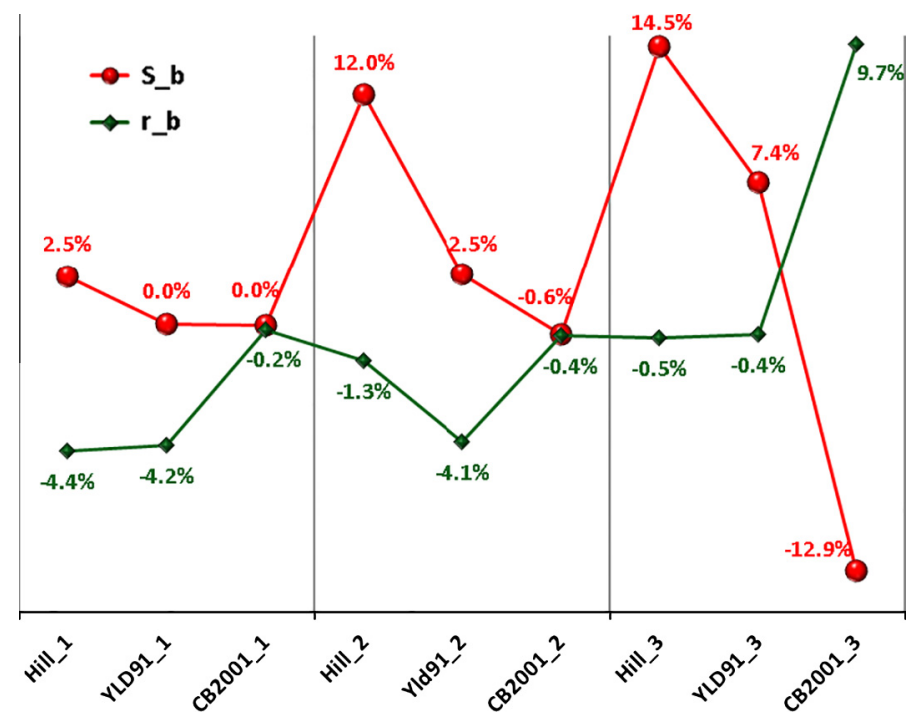

Fig. 4. Relative percent difference for balanced biaxial stress $\sigma_{b}$ and $r_{b}$-value for DC06 using the different yield criteria, as predicted by the three identification strategies.

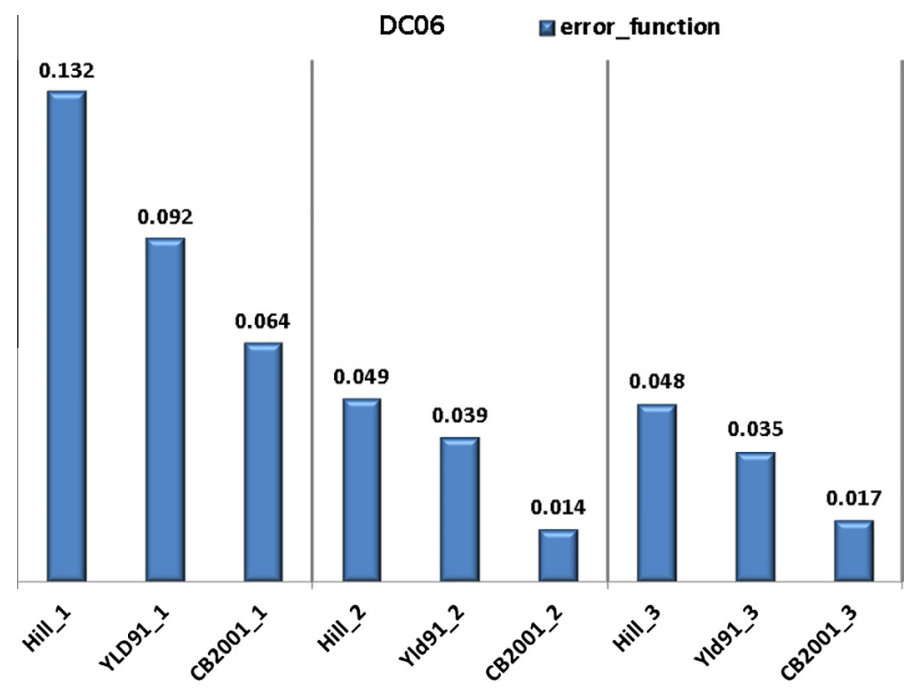

Fig. 5. Cost function obtained for the different yield criteria using the three identification strategies (DC06).

restarted several times from different random sets of parameters. It is observed that the same optimal solution is found, which is assumed to represent a global minimum. The results are labelled using the acronym for the yield criteria followed by the number corresponding to the identification strategy, i.e. YLD_X, were YLD refers to Hill, Yld91 or CB2001 and X can be assigned the value of 1, 2 or 3. For instance, Hill_1 corresponds to the Hill48 yield criterion identified using the first strategy.

\section{Analysis of the identification strategies results}

The anisotropy parameters obtained for the mild steel DC06, using the three identification strategies proposed, are listed in Table 5. For this material, there is a small difference between the anisotropy parameters obtained with the first and the second identification strategies, indicating a small effect of the shear stresses in the calibration procedure. The differences become more perceptible when biaxial data is removed, particularly for CB2001 yield criterion.

Fig. 1 depicts the predicted $r$-values for Hill48, Yld91 and CB2001 for the three strategies, showing that Hill48 and Yld91 give similar results, whatever the identification strategy selected. As expected, the results predicted by CB2001 are the closest to the experimental points. 
Table 6

Anisotropy parameters for AA6016-T4 obtained by the three strategies.

\begin{tabular}{|c|c|c|c|c|c|c|}
\hline Hill48 & $F$ & $G$ & $H$ & $N$ & & \\
\hline Hill_1 & 0.6456 & 0.6188 & 0.4389 & 1.3807 & & \\
\hline Hill_2 & 0.5664 & 0.5429 & 0.3850 & 1.2004 & & \\
\hline Hill_3 & 0.5591 & 0.5607 & 0.3886 & 1.2113 & & \\
\hline Yld91 & $c_{1}$ & $c_{2}$ & $c_{3}$ & $c_{6}$ & $m$ & \\
\hline Yld91_1 & 1.0572 & 1.0428 & 0.9444 & 0.9498 & 8 & \\
\hline Yld91_2 & 1.0257 & 1.0117 & 0.9162 & 0.9207 & 8 & \\
\hline Yld91_3 & 1.0184 & 1.0194 & 0.9165 & 0.9209 & 8 & \\
\hline \multicolumn{7}{|l|}{ CB2001 } \\
\hline \multirow[t]{4}{*}{ CB2001_1 } & $a_{1}$ & $a_{2}$ & $a_{3}$ & $a_{4}$ & $c$ & \\
\hline & 1.0086 & 1.2802 & 1.1868 & 1.0407 & 1.5882 & \\
\hline & $b_{1}$ & $b_{2}$ & $b_{3}$ & $b_{4}$ & $b_{5}$ & $b_{10}$ \\
\hline & 1.5449 & 1.1901 & 1.1114 & 1.2999 & 1.5739 & 1.6054 \\
\hline \multirow[t]{4}{*}{ CB2001_2 } & $a_{1}$ & $a_{2}$ & $a_{3}$ & $a_{4}$ & $c$ & \\
\hline & 0.7553 & 1.1337 & 1.1129 & 0.8497 & 0.2935 & \\
\hline & $b_{1}$ & $b_{2}$ & $b_{3}$ & $b_{4}$ & $b_{5}$ & $b_{10}$ \\
\hline & 1.7821 & -2.0689 & -1.5247 & -0.6334 & 1.1179 & 1.0937 \\
\hline \multirow[t]{4}{*}{ CB2001_3 } & $a_{1}$ & $a_{2}$ & $a_{3}$ & $a_{4}$ & $c$ & \\
\hline & 1.0182 & 1.1614 & 1.1791 & 0.8385 & 0.7658 & \\
\hline & $b_{1}$ & $b_{2}$ & $b_{3}$ & $b_{4}$ & $b_{5}$ & $b_{10}$ \\
\hline & -2.3508 & -1.9733 & 1.9697 & 2.3936 & 0.8269 & -0.2035 \\
\hline
\end{tabular}

Fig. 2 shows results for the three strategies, presenting the predicted tensile stresses along with experimental results for the plastic strain value $\bar{\varepsilon}^{\mathrm{pl}}=10 \%$. In this figure, and whenever considered relevant, the results obtained with the isotropic von Mises yield criterion are presented for comparison purposes. For the first strategy it is observed that the predicted directional tensile stresses are underestimated (relative to the experimental ones), with a similar trend for all yield criteria. However, the difference between the two most distant points (experimental and predicted values) in Fig. 2(a) does not exceed $10 \%$. The second and the third identification strategies lead to a better prediction of the experimental directional stress values, indicating that the shear stress values are contributing to the overall decrease in the mean value of the predicted tensile stress. This effect can be minimised by using a smaller weighting factor for the shear stress values [43]. As expected, the CB2001 yield criterion leads to a better prediction of the experimental directional tensile stress values.

Fig. 3 presents the initial yield stress surfaces, in the $\sigma_{1} v$ s. $\sigma_{2}$ space, as predicted using the three strategies. Fig. 3(a) shows that the three yield surfaces are almost superposed, thus the first identification strategy leads to a good agreement between the predicted initial yield surfaces and those defined by experimental points. The removal of the shear stress values from the calibration strategy has a small effect on the yield surface locus, as depicted in Fig. 3(b). However, the removal of the biaxial data has a strong impact on all yield criteria as shown in Fig. 3(c). In fact, CB2001 fails to predict the yield locus shape when only directional $r$-values and tensile stresses are used, leading to a yield surface quite similar to that of von Mises (see Fig. 3(c)). The relative error between predicted and experimental biaxial values is presented in Fig. 4, showing that when biaxial experimental data is used in the identification strategy, the CB2001 yield criterion leads to a relative error smaller than $1 \%$. However, if this data is removed from the input data set, this criterion presents the highest deviations. Moreover, Yld91 is able to predict biaxial values with an acceptable error (less than 5\%), when using biaxial data. When this data is removed the relative error slightly increases (less than $10 \%$ ).

Fig. 5 compares the cost function values attained at the end of each calibration strategy for each yield criterion. The values corresponding to Hill48 are always higher compared to the other yield criteria, emphasising that this quadratic yield criterion cannot attain predictions as accurate as those obtained by Yld91 and CB2001.

Table 6 presents the anisotropy parameters for the aluminium alloy AA6016-T4 obtained with the three identification strategies. For this material, Hill48 and Yld91 yield criteria lead to similar anisotropy parameters for the second and the third calibration strategies. The CB2001 anisotropy parameters are quite different for the three identification strategies.

Fig. 6 depicts the $r$-values obtained by the three yield criteria, for the three strategies. The $r$-values obtained by Hill 48 and Yld91 are practically superposed for the second and third strategy. Fig. 7 shows the directional yield stresses predicted by the three identification strategies for the yield criteria studied. The first strategy presents some deviation in the predicted mean tensile stress value, with Hill48 and Yld91 showing a similar trend. In fact, when the second and the third strategy are applied to those yield criteria, the predicted tensile stresses are also quite similar. Globally, the predicted average yield stress value is underestimated when the first strategy is applied, similarly to what was observed for DC06 steel. The yield surfaces in the $\sigma_{1} v$ s. $\sigma_{2}$ space are plotted in Fig. 8. The yield surfaces are quite similar to that of von Mises, particularly when the third 

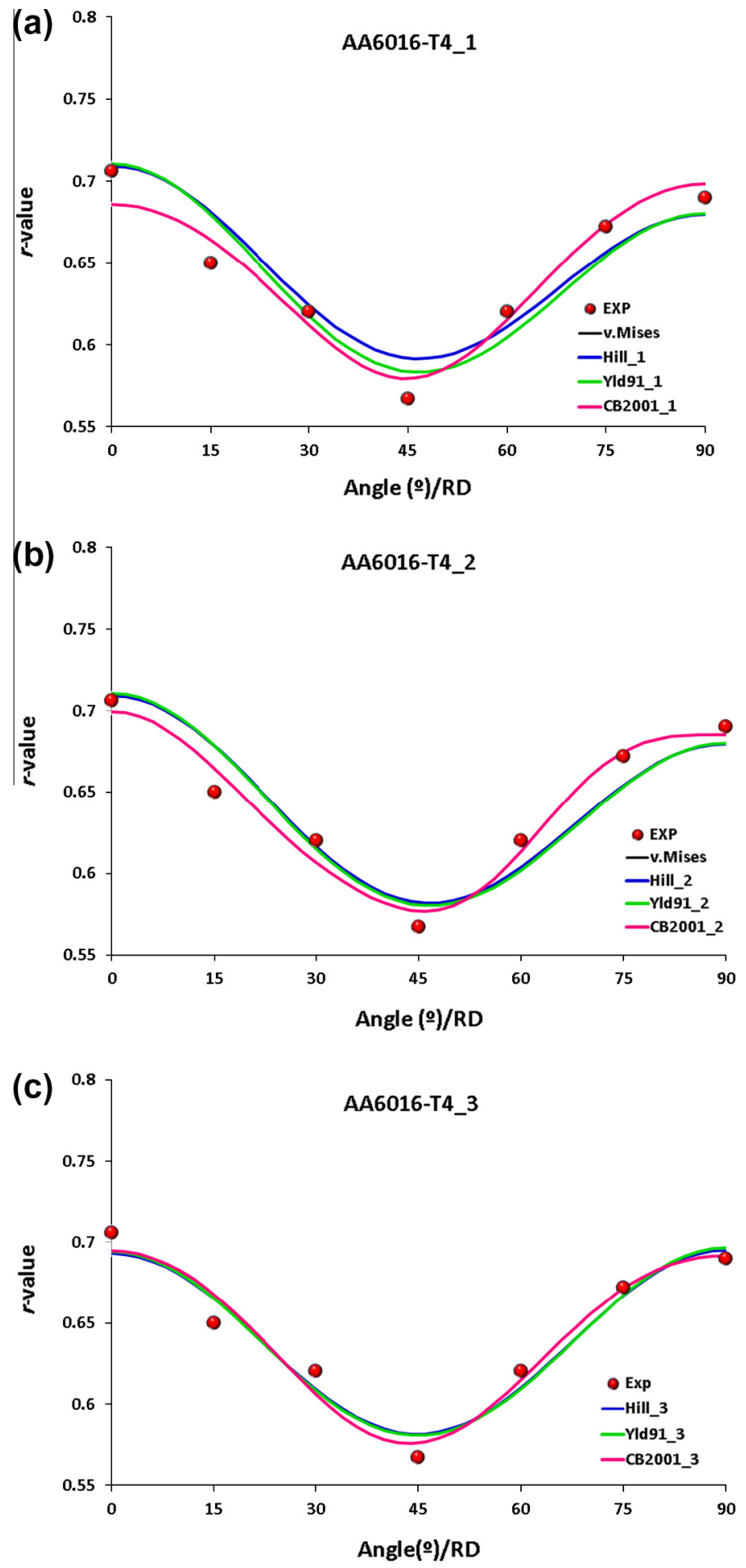

Fig. 6. Predicted $r$-values along with experimental data points for AA6016-T4 using the: (a) first strategy; (b) second strategy; and (c) third strategy.

identification strategy is employed. This seems to be a consequence of the low experimental planar anisotropy coefficient of AA6016-T4 material (see Table 4). It is also interesting to notice that the results obtained with Yld91 and CB2001, using the first identification strategy, are quite similar and lead to better predictions than with Hill48.

As for DC06 material, when neglecting the biaxial input data in the identification procedure, CB2001 does not accurately describe this loading mode. Fig. 9 shows the relative error for the biaxial data obtained with the different calibration strategies, for the yield criteria studied. Globally the differences for the biaxial stress values are lower than those found with DC06. 

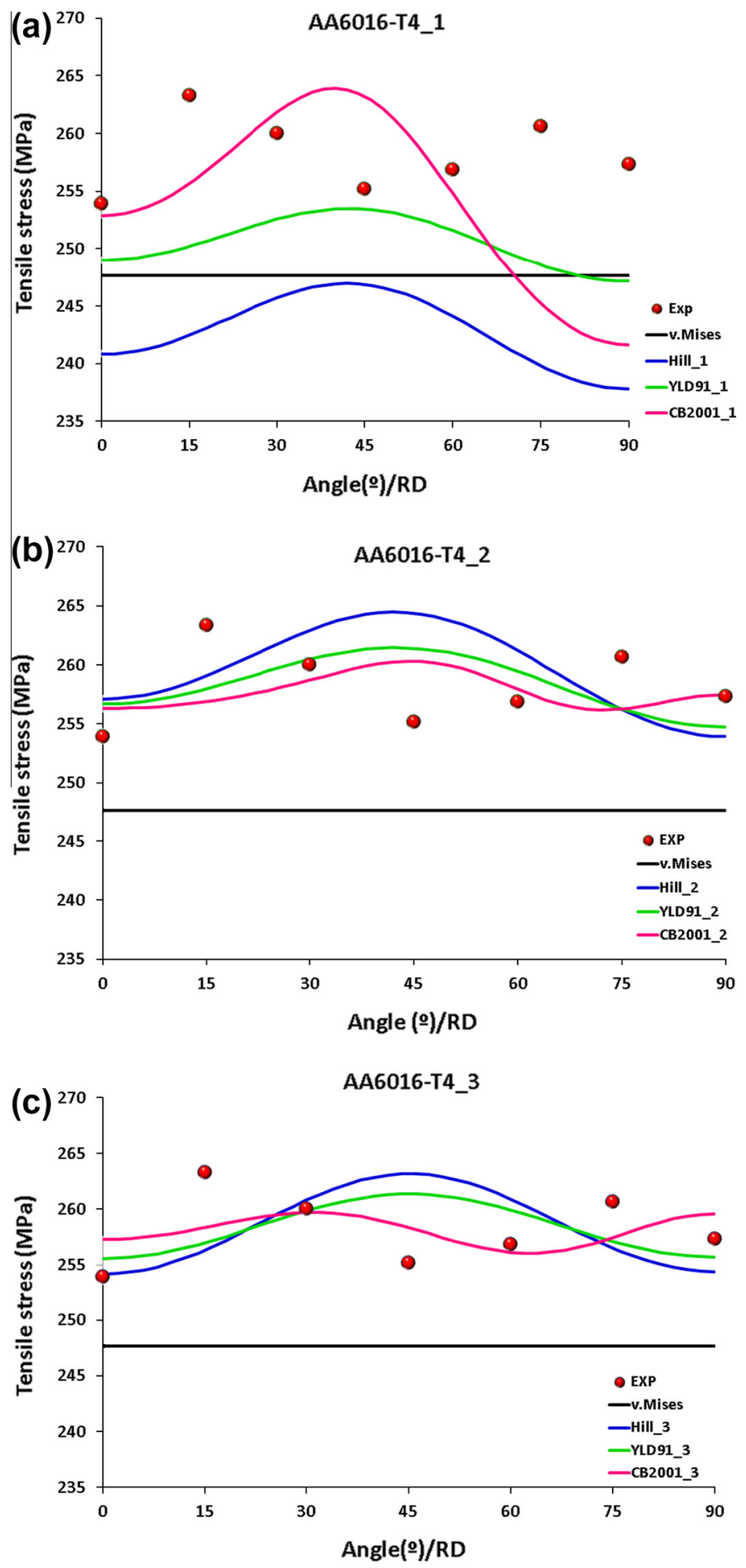

Fig. 7. Predicted tensile stresses along with experimental data points for AA6016-T4 using the: (a) first strategy; (b) second strategy; and (c) strategy.

Furthermore, the biaxial data predicted by Yld91 is close to the experimental values. In fact, the biaxial stress values predicted by Yld91 with the second and the third identification strategies are similar (i.e. relative error is $-0.2 \%$ ). However, for the CB2001 yield criterion, the relative error in the biaxial stress value changes from $1.0 \%$ to $-6.0 \%$, when the experimental balanced biaxial data is removed from the identification procedure. It seems that this consequence is not specific to CB2001, also being valid for other advanced yield criteria $[21,31,41]$. 


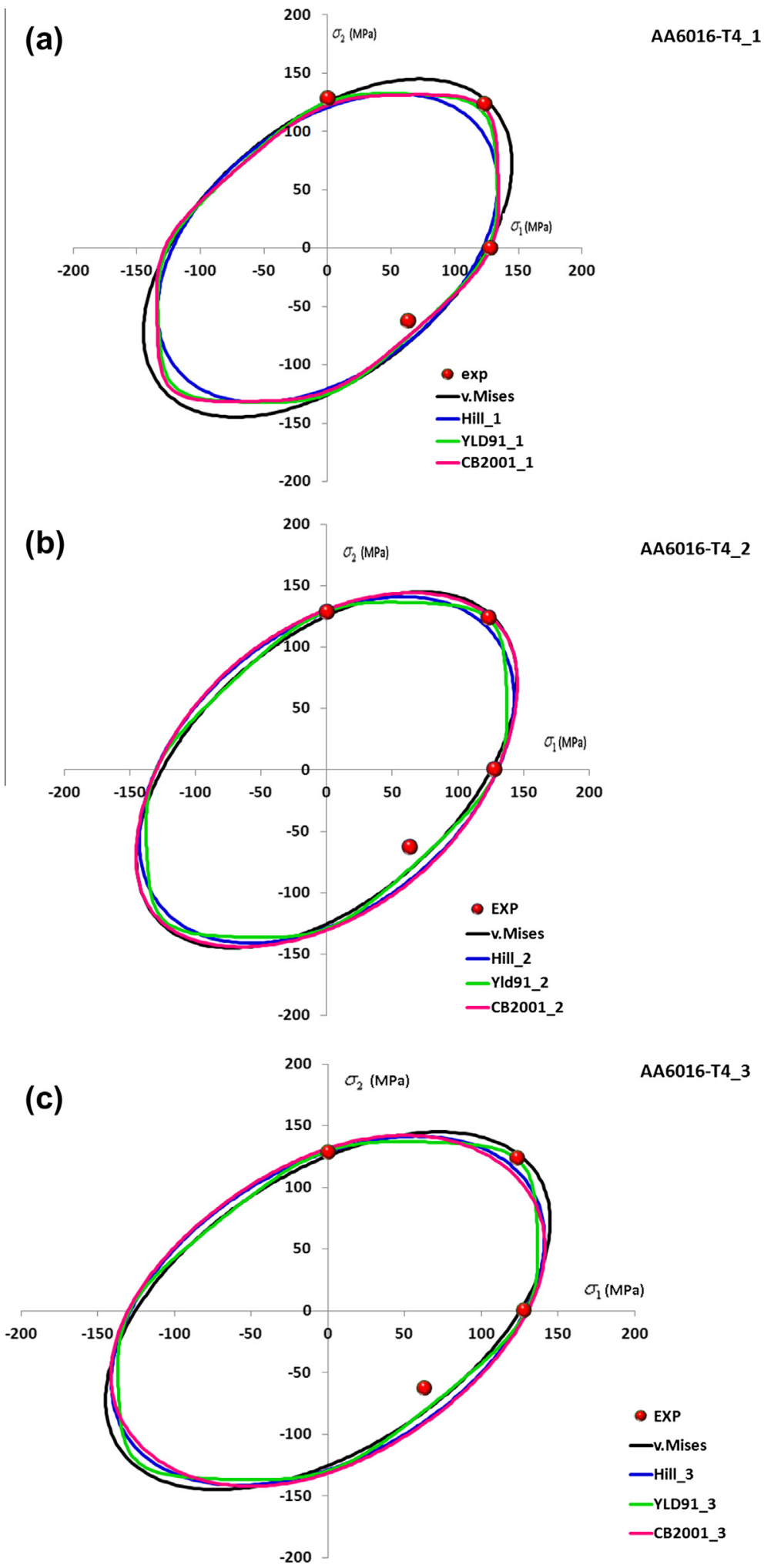

Fig. 8. Initial yield surfaces in the $\sigma_{1}$ vs. $\sigma_{2}$ space for AA6016-T4 using the: (a) first strategy; (b) second strategy; and (c) third strategy. 


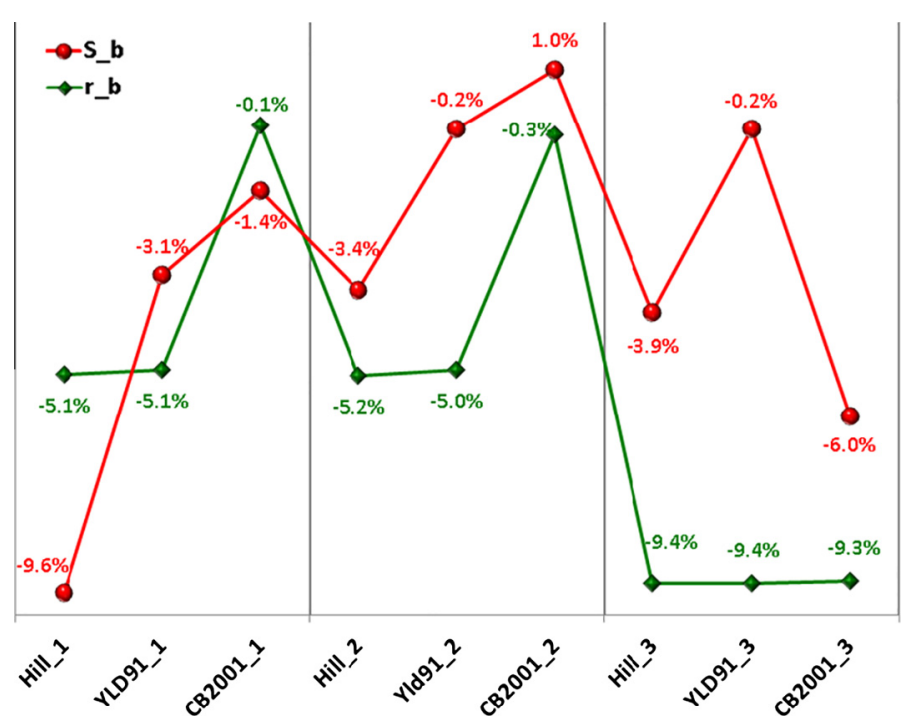

Fig. 9. Relative percent difference for balanced biaxial stress $\sigma_{b}$ and $r_{b}$-value for AA6016-T4 using the different yield criteria, as predicted by the three identification strategies.

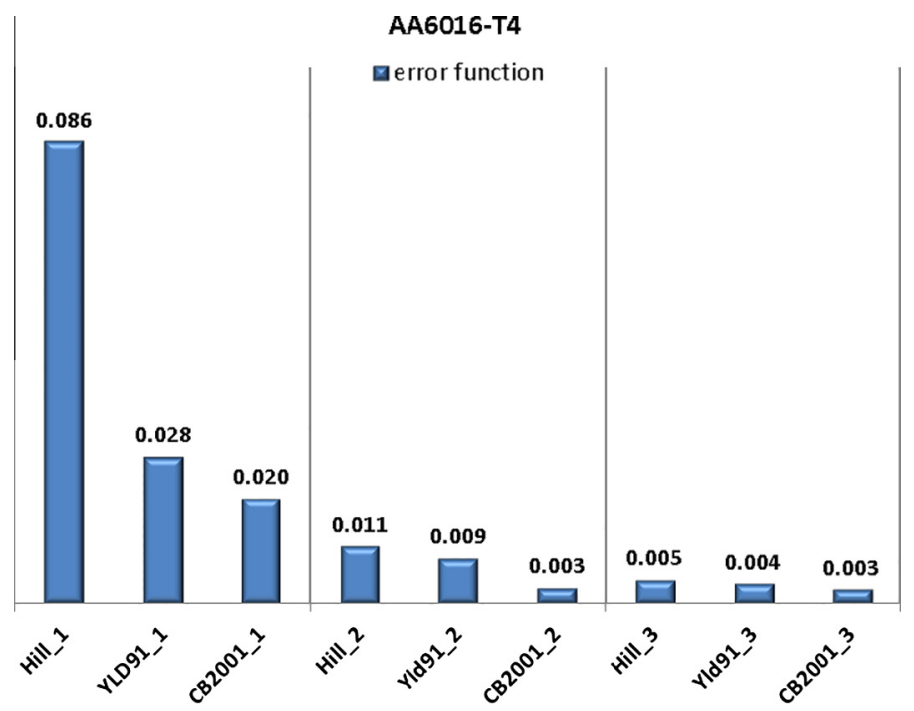

Fig. 10. Cost function obtained for the different yield criteria using the three identification strategies (AA6016-T4).

Fig. 10 shows the cost function values attained with the proposed calibration strategies, for the selected yield criteria. Analogous conclusions can be drawn for the aluminium alloy AA6016-T4 as for the mild steel DC06. However, the cost function values are always lower than those observed for DC06. This seems to result from the fact that the DC06 $r$-values present a more relevant anisotropic behaviour than those of AA6016-T4 (see Tables 3 and 4).

Globally, the use of all available experimental data to identify the anisotropy parameters of yield criteria leads to a good agreement between predicted and experimental results, for both materials. However, directional tensile stresses are poorly predicted when also considering shear stresses in the calibration strategy, since they appear to contribute to a decrease in the mean predicted tensile stress value. Thus, by removing the shear stresses from the identification strategy, a better description of the directional tensile stresses was obtained. One can also observe that excluding biaxial input data from the calibration of the CB2001 yield surface leads to inaccurate results, particularly for the mild steel. The CB2001 leads to more accurate results than those predicted by Hill48 or Yld91 yield criteria, as long as adequate experimental input data is available. Yld91 seems to reasonably predict biaxial stress values, whenever this experimental data is missing from the 
Table 7

Experimental and artificial data (obtained with Yld91) used to calibrate CB2001 for DC06.

\begin{tabular}{cllll}
\hline Angle from RD & $r$-value & Uniaxial Stress at $\bar{\varepsilon}^{\mathrm{pl}}=10 \%$ & Biaxial stress $(\mathrm{MPa})$ & Biaxial $r$-value \\
\hline 0 & $\mathbf{2 . 5 3}$ & $\mathbf{2 9 2 . 8}$ & \\
15 & 2.35 & 291.0 & \\
30 & 1.99 & 304.3 & \\
45 & $\mathbf{1 . 8 4}$ & $\mathbf{3 0 3 . 5}$ & \\
60 & 2.07 & 305.7 & \\
75 & 2.50 & 293.2 & \\
90 & $\mathbf{2 . 7 2}$ & $\mathbf{2 8 7 . 5}$ & \\
\hline
\end{tabular}

Table 8

Experimental and artificial data (obtained with Yld91) used to calibrate CB2001 for AA6016-T4.

\begin{tabular}{cllll}
\hline Angle from RD & $r$-value & Uniaxial Stress at $\bar{\varepsilon}^{\mathrm{pl}}=10 \%$ & Biaxial stress $(\mathrm{MPa})$ & 122.1 \\
\hline 0 & $\mathbf{0 . 7 1}$ & $\mathbf{2 5 3 . 9}$ & \\
15 & 0.67 & 255.0 & \\
30 & 0.60 & 258.2 & \\
45 & $\mathbf{0 . 5 7}$ & $\mathbf{2 5 5 . 2}$ & \\
60 & 0.60 & 257.8 & \\
75 & 0.66 & 254.4 & \\
90 & $\mathbf{0 . 6 9}$ & $\mathbf{2 5 7 . 3}$ & \\
\hline
\end{tabular}

Table 9

Anisotropy parameters obtained for CB2001 with experimental and artificial data for DC06.

\begin{tabular}{llllll}
\hline CB2001_min & $a_{1}$ & $a_{2}$ & $a_{3}$ & $a_{4}$ & $c$ \\
\hline & 1.2028 & 0.9574 & 0.9687 & 1.0078 & $b_{4}$ \\
& $b_{1}$ & $b_{2}$ & $b_{3}$ & $b_{10}$ \\
\cline { 2 - 5 } & 0.4773 & 2.2131 & 2.3047 & 0.2087 & 0.7125 \\
\hline
\end{tabular}

experimental set. Therefore, Yld91 biaxial predictions could be used as an alternative solution to provide meaningful data, when no experimental data is available. The possibility of using artificial data combined with a reduced number of experimental tests to calibrate CB2001 is investigated in the next section. This concept may look like looped reasoning since, instead of relying on full experimental data sets, artificial data is adopted, derived from less flexible yield criteria, and calibrated using a smaller amount of experimental data. The idea is to show that the selection of the yield criterion should not necessarily be determined based on the amount of available experimental data. In particular, advanced yield criteria should not be excluded when biaxial experimental data is unavailable.

\subsection{Identification of CB2001 using a reduced set of experimental input data}

CB2001 yield criterion involves 11 anisotropy parameters, requiring a high amount of input data obtained from mechanical tests carried out in different loading modes, particularly in the balanced biaxial strain path [13,30]. In fact, the necessary conventional input data used to accurately calibrate CB2001 involves 7 directional tensile stresses, 7 directional $r$-values, the balanced biaxial yield stress and the balanced biaxial strain ratio $r_{b}$-value [44]. However, this latter type of test is not usually available in industrial or research laboratories. On the other hand, as shown in the previous section, this yield criterion allows an improved description of directional tensile stresses and $r$-values. Thus, the idea is to try to explore the advantages of more flexible yield criteria, whenever a limited set of experimental data is available. To make the proof of concept, an identification procedure for CB2001 is proposed using the minimum amount of experimental data, i.e. the results obtained from three uniaxial tensile tests. However, under this assumption, the predefined minimisation procedure is mathematically under-constrained. Hence, it is necessary to resort to "virtual data", obtained with a simpler yield criterion.

In this section, it is assumed that the available experimental data consists of uniaxial tensile tests (i.e. three yield stresses and three $r$-values), performed at three orientations, $0^{\circ}, 45^{\circ}$ and $90^{\circ}$, to RD. This number and type of experimental data is considered as the minimum required for the mechanical characterisation of anisotropic metal sheets. However, to identify the 11 anisotropy parameters of the CB2001 criterion, at least 11 experimental values are required [33]. The missing input data is the additional directional tensile stresses and $r$-values along with biaxial values, which are generated using the Yld91 
Table 10

Anisotropy parameters obtained for CB2001 with experimental and artificial data for AA6016-T4.

\begin{tabular}{|c|c|c|c|c|c|c|}
\hline CB2001_min & $a_{1}$ & $a_{2}$ & $a_{3}$ & $a_{4}$ & $c$ & \\
\hline & 0.8427 & 1.0343 & 1.4461 & 0.8273 & 0.1364 & \\
\hline & $b_{1}$ & $b_{2}$ & $b_{3}$ & $b_{4}$ & $b_{5}$ & $b_{10}$ \\
\hline & -3.4430 & -7.7347 & -2.2980 & 4.1896 & 1.3882 & -0.3860 \\
\hline
\end{tabular}

yield criterion. These material properties are considered to be artificial data but are essential for the parameter identification procedure. Therefore, the first step of the identification procedure consists of the calibration of the Yld91 criterion using input data gathered from the three tensile tests carried out at orientations of $0^{\circ}, 45^{\circ}$ and $90^{\circ}$ to $\mathrm{RD}$.

Yld91 is not flexible enough to simultaneously accurately reproduce $r$-values and directional tensile stresses [28]. Therefore, Yld91 calibration is performed using the same cost function (see Eq. (17)), but considering two different combinations of the weighting factors assigned to the experimental data. To determine the artificial yield stress values, high weighting factors are assigned to the experimental yield stresses, while low weighting factors are defined for the $r$-values. The calibration used to predict the $r$-values inverts the weighting factors. The values of 1.0 and 0.001 are used as high and low weighting factors, respectively.

Table 7 lists DC06 material properties comprising the "effective experimental data" corresponding to the directions $0^{\circ}$, $45^{\circ}$ and $90^{\circ}$, with respect to RD, and the artificial data for the other orientations, as predicted by the Yld91. The material data set used to calibrate CB2001 for AA6016-T4 is summarised in Table 8. The biaxial values, $\sigma_{b}$ and $r_{b}$, predicted by Yld91 are close to those attained using the set of 14 uniaxial tensile test results (see Figs. 4 and 9). In fact, for DC06, the relative difference is about 7.6\% and 3\% for the biaxial stress and $r_{b}$, respectively. For AA6016-T4, the relative difference for $\sigma_{b}$ and $r_{b}$ is $-1 \%$ and $-7.2 \%$, respectively.

The proposed approach based on Yld91 predictions can generate artificial biaxial yield stress values $\sigma_{b}$ and $r_{b}$ that do not properly describe the material's behaviour. These erroneous predictions will affect the estimate of the material's anisotropic behaviour when using more advanced models, particularly near the biaxial loading point. However, since more advanced models are more flexible, it is expected to obtain improved descriptions for other loading points, when compared with the Yld91. Moreover, more flexible yield criteria involving five parameters, such as Karafillis and Boyce (KB93) yield criterion, can be used to generate the artificial data. Nevertheless, it is not possible to know in advance how well the artificial data reproduces the material behaviour. Thus, in the worst case the numerical predictions will be similar to those obtained with the less flexible yield criteria.

The CB2001 anisotropy parameters determined using the proposed identification strategy, referred to as "CB2001_min", are listed in Tables 9 and 10, for DC06 and AA6016-T4, respectively. Fig. 11(a) and (b) present the $r$-values and the uniaxial tensile stresses, as predicted by the CB2001 yield criterion for DC06, along with experimental and "artificial input data". The $r$-values are well adjusted by the CB2001, whereas the tensile stresses are slightly different from the target points, with a maximum deviation of about 3\%. The results for the initial predicted yield surfaces are shown in Fig. 11(c), along with the values for the experimental initial yield stresses. The Yld91 yield surface used only 6 elements of input data for calibration, with higher weighting factors for the stress values. Both Yld91 and CB2001 provide a good approximation to the experimental results.

Fig. 12(a) and (b) present the $r$-values and the uniaxial tensile stresses, as predicted by the CB2001 yield criterion for AA6016-T4, along with experimental and "artificial input data". Looking at the $r$-values, the conclusions for this material are similar to those mentioned for DC06. The maximum deviation between tensile stresses predicted by CB2001 and the experimental and artificial data is $1 \%$ (see Fig. 12(b)). Fig. 12(c) presents the initial yield surface along with experimental results, for both Yld91 and CB2001. For this material, there is a good agreement between Yld91 and CB2001, mainly in the first quadrant of the yield locus. Nevertheless, it is important to highlight that calibration of the Yld91 surface used only 6 values of input data with higher weighting factors for the stress values.

\section{Experimental validation of identification strategies using the cross die test}

Fig. 13 shows a cup drawn using the cross die tool shape used in this study. This tooling device has been designed to reproduce most of the industrial strain paths, i.e. uniaxial tensile, plane strain, shear and biaxial stretching. Hence, it is used in the automotive industry to investigate the effect of the hardening laws and yield criteria description on the accuracy of FE simulation of industrial parts. In the current study, the punch force evolution and the thickness distributions were used to analyse the proposed identification strategies. The thickness distribution was measured along three directions from the centre of the deformed part: the rolling direction or median direction (OX), the diagonal direction (OXY) and the transverse direction (OY) [45,46], as shown in Fig. 13. However, since the analysis of the thickness evolutions along RD and TD directions leads to similar results, only the thickness measured along the median and diagonal paths are reported hereafter. The blank is a square metal sheet of $250 \times 250 \mathrm{~mm}^{2}$. The DC06 cup is produced considering a blank-holder force of $290 \mathrm{kN}$ while 

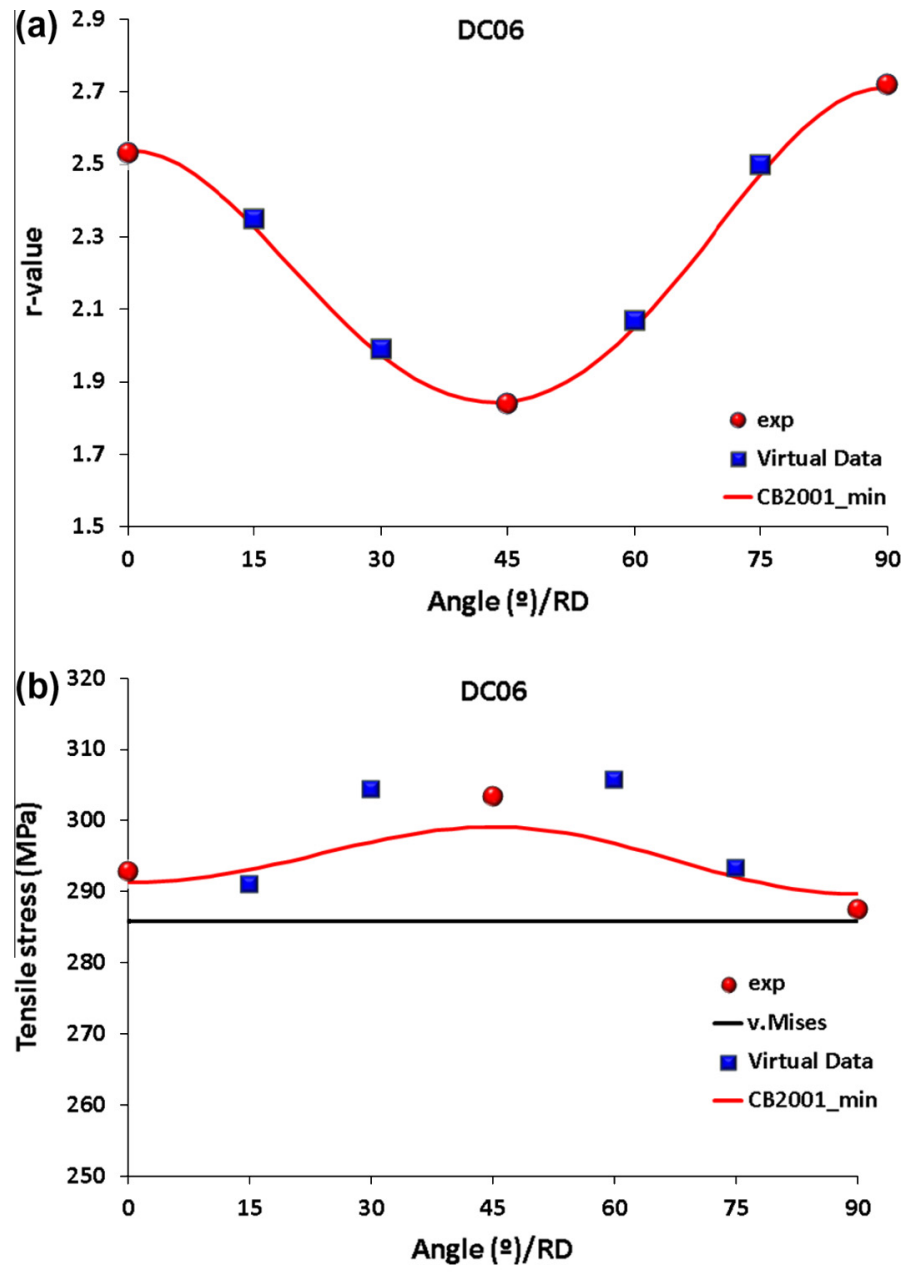

(c)

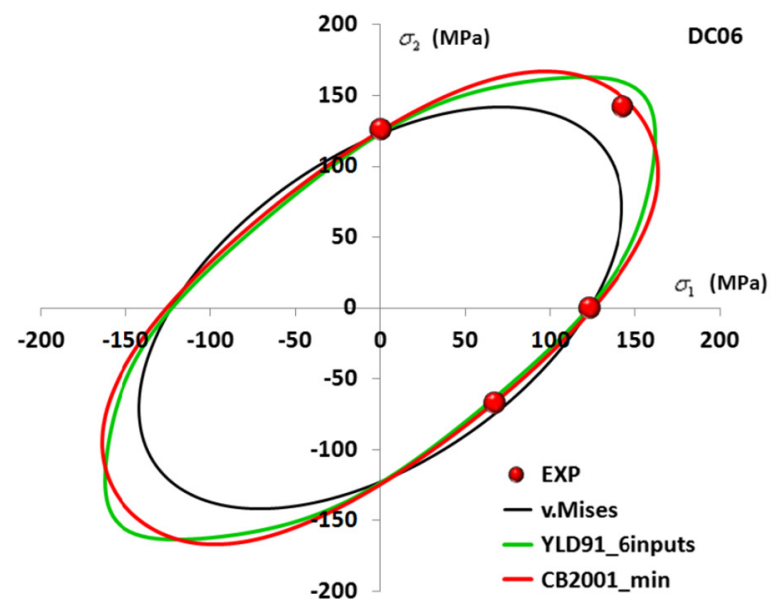

Fig. 11. Predicted $v s$. experimental: (a) $r$-values; (b) tensile stresses at equivalent plastic strain of $10 \%$; and (c) initial yield surfaces in $\sigma_{1}, \sigma_{2}$ space, for DC06.

for AA6016-T4 a blank-holder force of $60 \mathrm{kN}$ is used. For both materials Coulomb's friction coefficient is set at 0.03 for all contact areas.

The FE simulation of this forming test was carried out using the in-house code DD3IMP [47]. The blank was discretized using 8-node hexahedron solid finite elements with selective reduction integration. Based on geometrical and material 

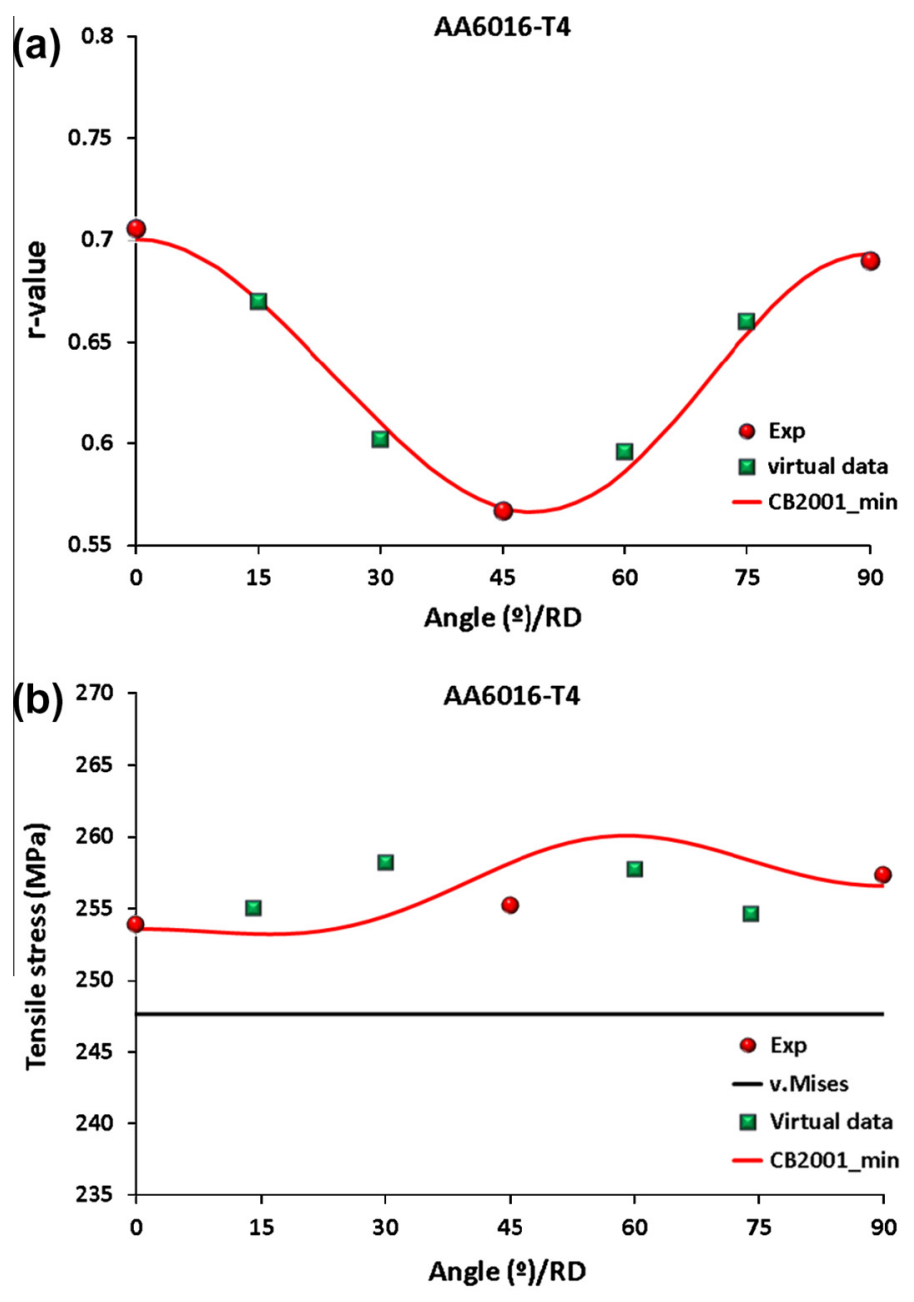

(c)

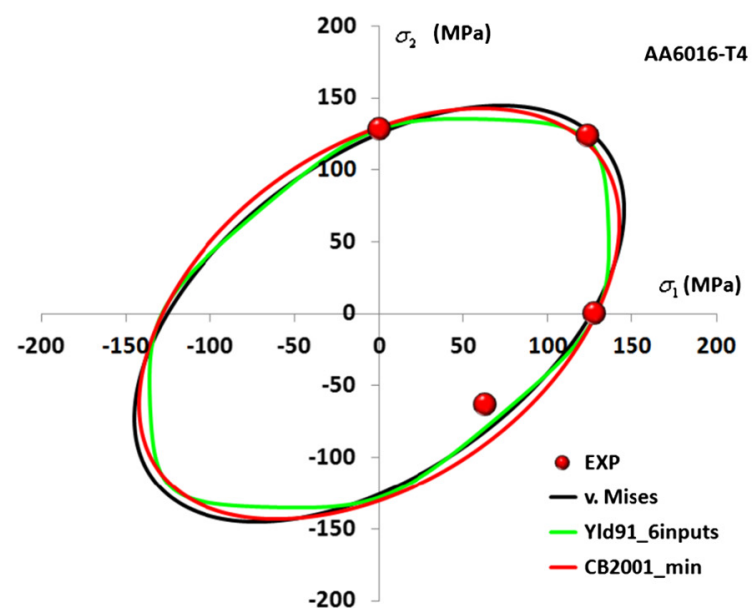

Fig. 12. Predicted vs. experimental: (a) $r$-values; (b) tensile stresses at equivalent plastic strain of $10 \%$; and (c) initial yield surfaces in $\sigma_{1}, \sigma_{2}$ space, for AA6016-T4.

symmetries, only one fourth of the blank is modelled using 62 by 62 in-plane finite elements, with two layers of elements throughout the thickness.

Fig. 14 shows the predicted punch force evolution, for all yield criteria, using the proposed identification strategies for both materials. The punch force is a global variable and therefore is roughly insensitive to the constitutive parameter 


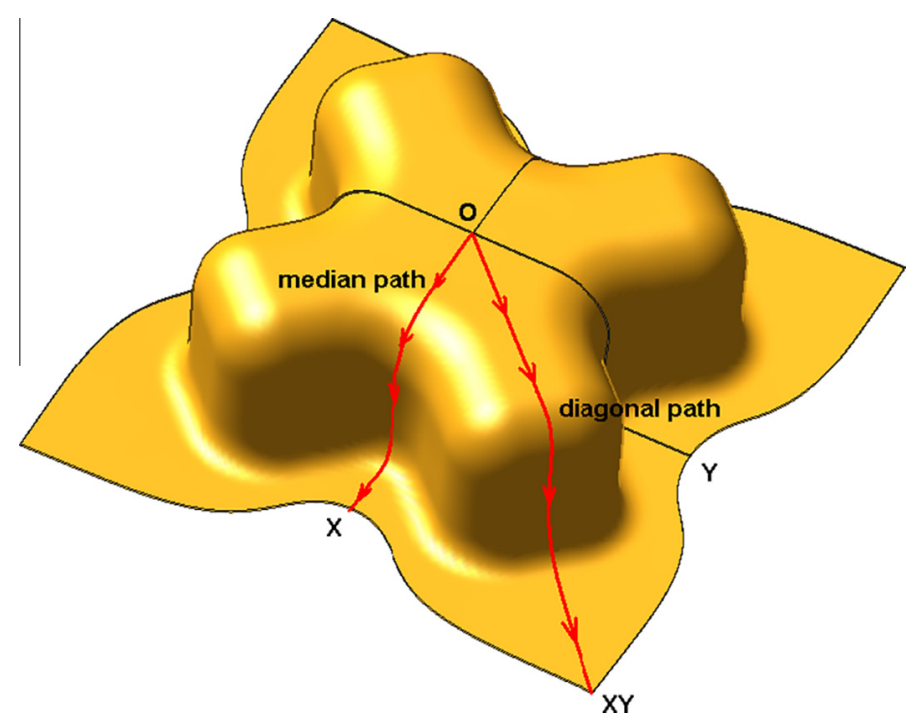

Fig. 13. Deep drawn cup obtained by the cross die tool and thickness measurement paths.

identification strategies and yield criteria, with the range between lower and upper punch force predictions reaching about $10 \%$ for both materials. For AA6016-T4 a decrease in the punch force is observed for a drawn depth of about $55 \mathrm{~mm}$, except for the simulation performed with the von Mises yield criterion. This decrease indicates the occurrence of necking, which is mainly observed for material points subjected to biaxial strain and plane strain paths during the forming process, highlighting the importance of the yield criterion in necking prediction. For DC06 steel, no necking occurs until the punch stroke reaches $60 \mathrm{~mm}$. Unfortunately, no experimental results for punch force evolution were available for either of the materials.

Figs. 15-17 show the experimental and predicted thickness evolution along the median and diagonal paths for DC06 steel, following a punch stroke of $30 \mathrm{~mm}, 40 \mathrm{~mm}$ and $60 \mathrm{~mm}$, respectively. The predicted results show a non-uniform thickness distribution and thinning in particular zones, along both paths. According to the FE results, along the median path, most thinning occurs under the punch fillet radius, where the material points are subjected to a plane strain state. The diagonal direction presents two points with similar thinning. The first is also located under the punch fillet radius where the material points are subjected to a biaxial stress state. The second thinning location is observed in the vertical wall of the part, for material points subjected to a uniaxial tensile stress state. Nonetheless, thinning in the diagonal direction is less critical than the one observed along the median path. The predicted thicknesses are dictated by the observed differences between the initial yield surfaces for the different calibration strategies. Globally, thickness predictions are less influenced by the identification strategy selected for Hill48 and Yld91 yield criteria. However, the calibration strategy strongly influences the thickness predictions attained with CB2001. For this material, lower thickness values are predicted by both von Mises and CB2001, as identified using the third calibration strategy (CB2001_3), since the yield surfaces of these two models are quite similar (see Fig. 3(c)).

For a punch stroke of $30 \mathrm{~mm}$ (see Fig. 15), using CB2001 and selecting the first identification strategy (CB2001_1) provides a good level of agreement between the experimental and predicted thickness distribution. Moreover, the thickness evolution predicted by CB2001 using the minimum amount of experimental data (CB2001_min) is quite similar to experimental measurements, along both paths. In fact, the results obtained are quite similar to those obtained with Yld91 using the third strategy (Yld91_3), a result which stems from the similar predictions for the biaxial strain paths. Moreover, the thickness evolution is better predicted by CB2001_min than by CB2001_3, highlighting the importance of the biaxial values in the identification procedure. For a punch stroke of $40 \mathrm{~mm}$ (see Fig. 16), numerical results deviate from the experimental data, along the median path, although along the diagonal path they show good agreement. This discrepancy is also observed for a punch stroke of $60 \mathrm{~mm}$ (see Fig. 17).

Turning to AA6016-T4, Figs. 18 and 19 present the predicted thickness evolutions along both median and diagonal paths for a punch stroke of $30 \mathrm{~mm}$ and $40 \mathrm{~mm}$, respectively. Although there is no experimental data available for this material, the predicted results are shown, since they enable better understanding of the influence of the anisotropy parameter identification strategy adopted. As with DC06, the thickness evolutions obtained with Hill48 and Yld91 present a negligible influence of the identification strategy. However, the calibration strategy used to identify the anisotropy parameters of CB2001 strongly dictates the thickness distribution. Contrary to DC06, lower thinning prediction is obtained when using von Mises's yield criterion for AA6016-T4, since in this case the normal anisotropy coefficient is lower than 1.0 (see Table 4). When the third identification strategy is applied, lower thickness values are predicted by Hill48 and CB2001. Globally, a smaller scattering is observed in the thickness distributions predicted by the different yield criteria and identification strategies, when compared to the thickness distributions obtained for DC06, particularly along the median direction. This is certainly related to the 

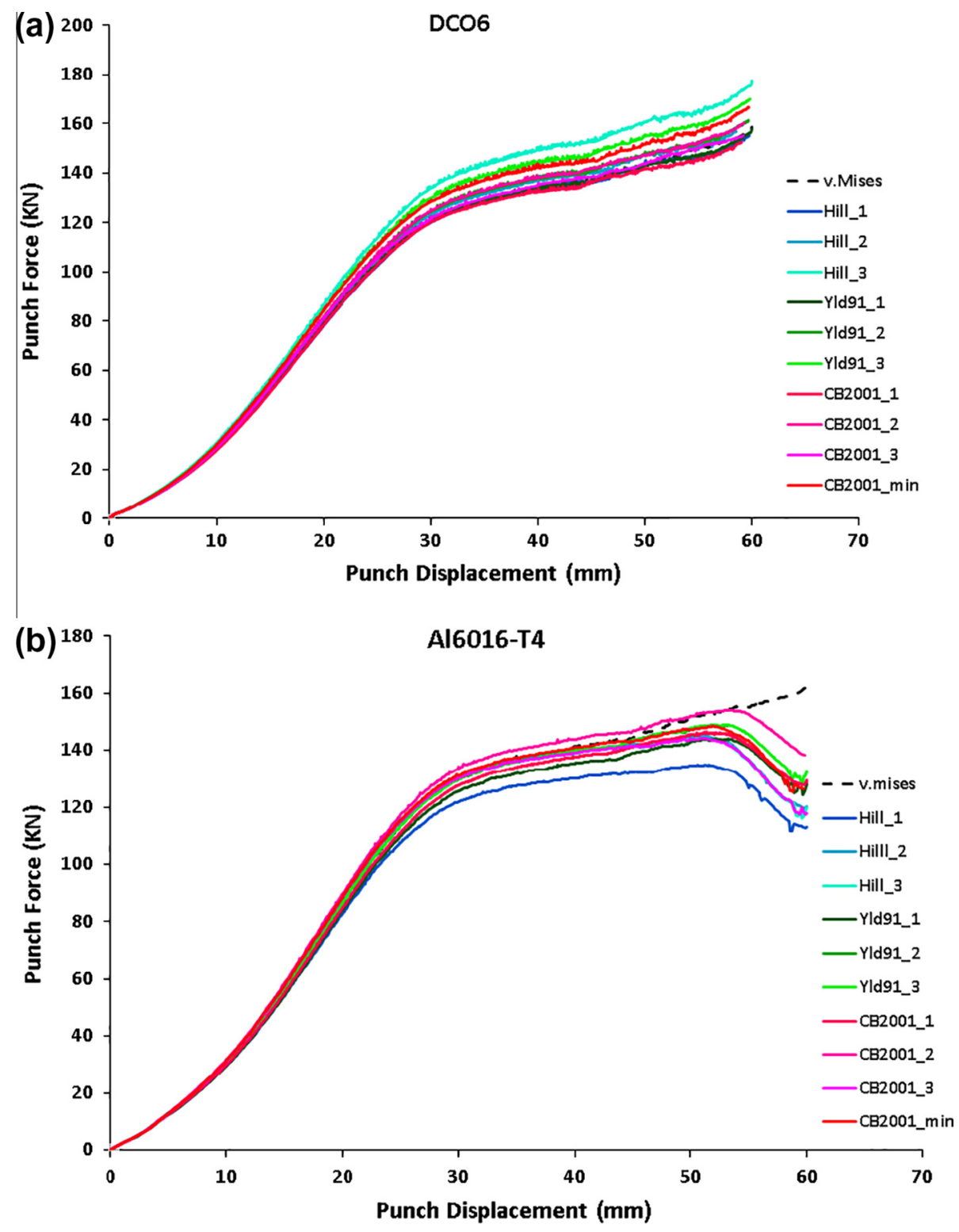

Fig. 14. Punch force vs. displacement for: (a) DC06 and (b) AA6016-T4.

almost similar initial yield surfaces obtained for AA6016-T4 (see Fig. 8). Results for the thickness evolution predicted by CB2001 using the calibration strategy with the minimum amount of experimental data (CB2001_min), are close to those obtained by CB2001 using the second identification strategy (CB2001_2). Since the choice of identification strategy makes little difference to the identification of the Yld91 criterion, the results are all quite similar to those predicted when using CB2001_min.

In brief, the number and type of experimental results used in the identification procedure is particularly important for materials presenting a higher value of the planar anisotropy coefficient. In case of materials presenting a low value of the planar anisotropy coefficient, it may be more important to evaluate the experimental biaxial values, particularly for components with pronounced biaxial stress states, as is the case of the diagonal path of the cross tool die.

To understand the discrepancy observed for DC06 steel between predicted and experimental thickness evolutions along the median path, for different punch strokes, the loading modes of material points located along both the median and diagonal paths were analysed. Additionally, studying a local variable enables an improved understanding of the thickness evolutions predicted by each yield criterion. The loading mode is calculated using the strain ratio $\beta$, defined by the ratio between the minor and major principal strains in the sheet plane, for a given point on the material (a node in the FE model). Fig. 20(a) 

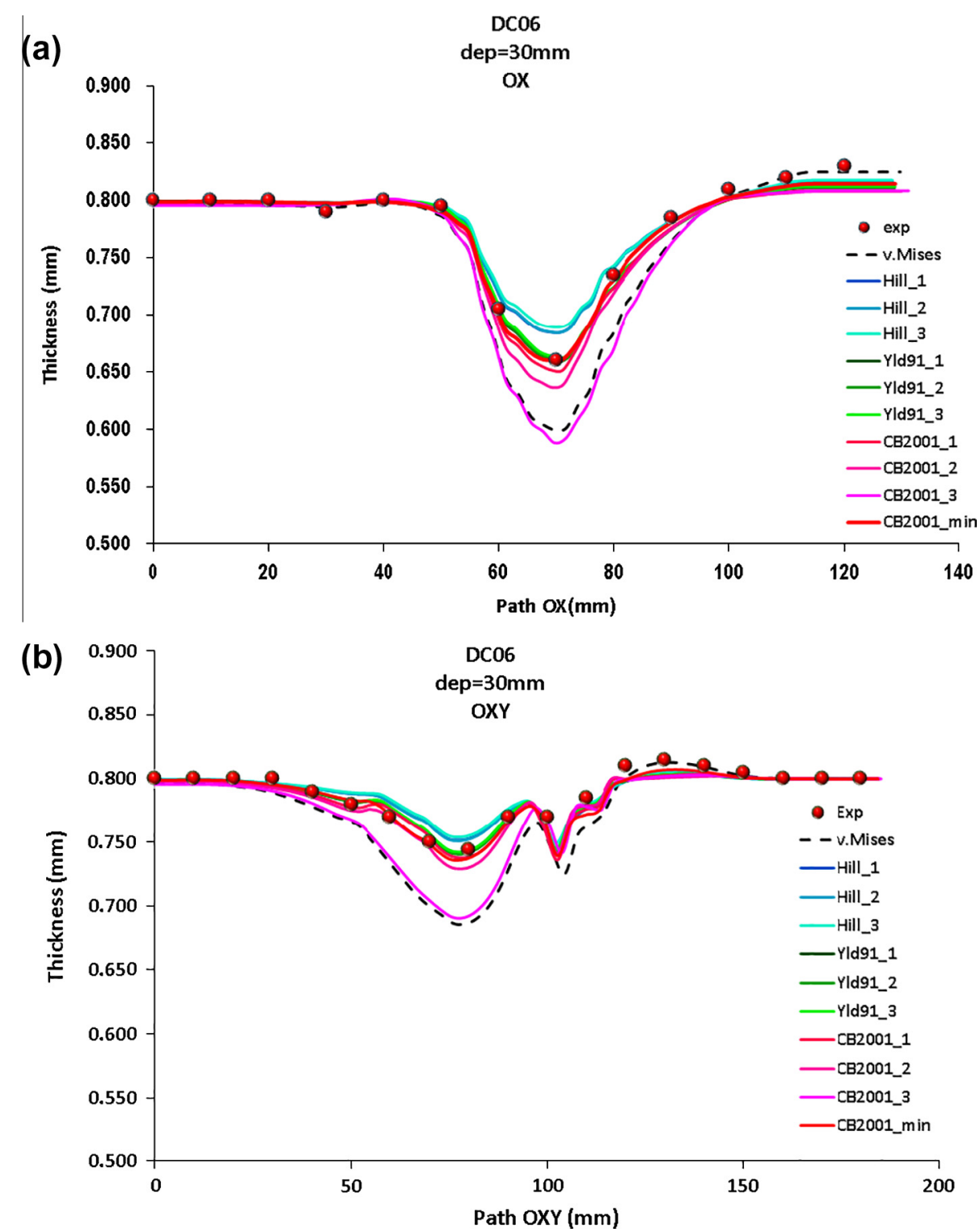

Fig. 15. Thickness evolution for DC06 for a punch stroke of $30 \mathrm{~mm}$, along the: (a) median path OX and (b) diagonal path OXY.

and (b) show the loading modes as predicted by the CB2001_min and the von Mises yield criteria, for material points located along the median and the diagonal paths, respectively. The nodes located in the centre of the cup correspond to the higher numbers. The strain paths related to equibiaxial strain $(\beta=1.0)$, plane strain $(\beta=0.0)$, uniaxial tensile stress $(\beta=-0.5)$ and shear $(\beta=-1.0)$, for an isotropic material, are used as reference values. Along the median path (see Fig. 20(a)), the strain state varies with the increase of the punch displacement. For instance, node 40 (corresponding to a material point under the punch fillet radius) is located close to a plane strain state for a punch stroke of $30 \mathrm{~mm}$, then close to a biaxial strain state for a punch displacement of $40 \mathrm{~mm}$, and finally, close to a uniaxial tensile state for a drawn depth of $60 \mathrm{~mm}$. On the contrary, the material points located along the diagonal path undergo almost the same loading mode for the three punch displacement levels, as shown in Fig. 20(b). Although not shown here, similar observations were made for both the Hill48 and Yld91 yield criteria. Thus, the strain state of a material point during the deformation process is mainly influenced by the tool's shape and the punch displacement. However, the strain ratio and the thickness evolutions are dictated by the normal to the yield surface, which is influenced by the yield criterion and the identification strategy adopted. Thus, since the predicted yield surfaces for the different yield criteria and identification strategies are more similar for the aluminium alloy, a smaller scattering of the thickness distributions is predicted for AA6016-T4 compared to DC06. 

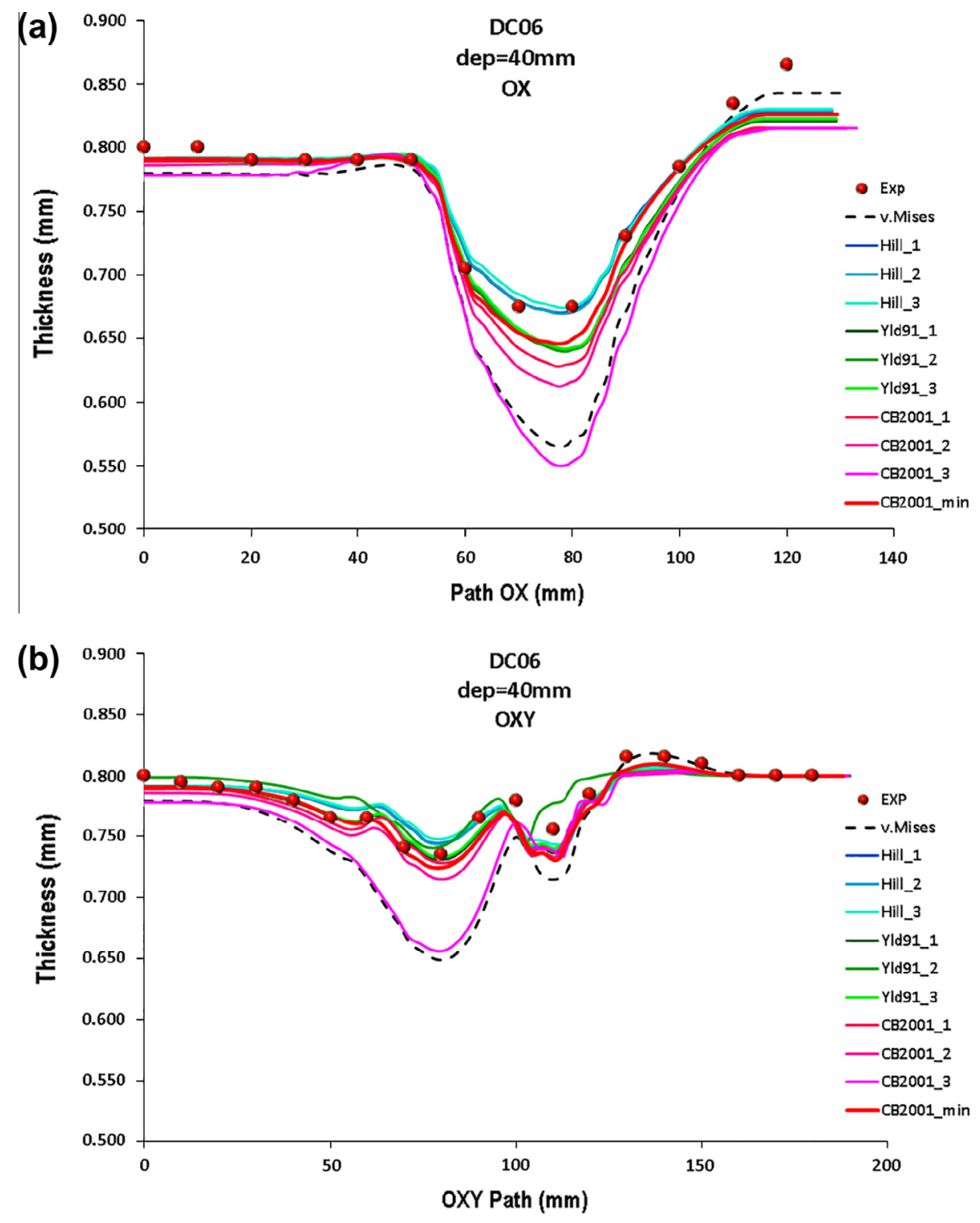

Fig. 16. Thickness evolution for DCO6 for a punch stroke of $40 \mathrm{~mm}$, along the: (a) median path OX and (b) diagonal path OXY.

Fig. 21(a) shows the location of the chosen nodes on the cross shaped formed cup. Nodes 40 and 1737 correspond to material points located under the punch fillet radius and are the first points subjected to thinning along the median and diagonal paths, respectively. Nodes 29 and 2233 correspond to material points located on the vertical wall of the part and nodes 9 and 2729 to material points that are located on the die fillet radius, along the median and diagonal directions, respectively. Fig. 21(b) shows the loading modes of these material points, with a punch stroke between $5 \mathrm{~mm}$ and $60 \mathrm{~mm}$, obtained with the CB2001 criterion using the identification strategy based on the minimum number of experimental results (CB2001_min). For instance, node 40 starts to deform close to a plane strain state, being close to a biaxial strain state for a punch displacement of $40 \mathrm{~mm}$ and close to a uniaxial tensile state for a displacement of $60 \mathrm{~mm}$. However, the corresponding node along the diagonal path (i.e. node 1737) undergoes a biaxial strain mode from the beginning of loading until the end of the forming process. Although not shown here, similar strain paths were obtained for the AA6016-T4 aluminium alloy. The material points located along the diagonal path tend to exhibit almost monotonous strain paths. Therefore, the differences observed in the thickness evolutions for different drawing depths mainly reflect the difference in the normal to the yield surface for those specific paths, as predicted by the different yield criteria, combined with the different identification strategies. However, material points located along the median path tend to present strain path changes from biaxial strain state to uniaxial tensile state (node 40) or shear to uniaxial tensile strain (node 9). Therefore, the thickness evolutions along the median path reflect the difference in the normal to the yield surface for all those strain states. This seems to be the reason behind the 

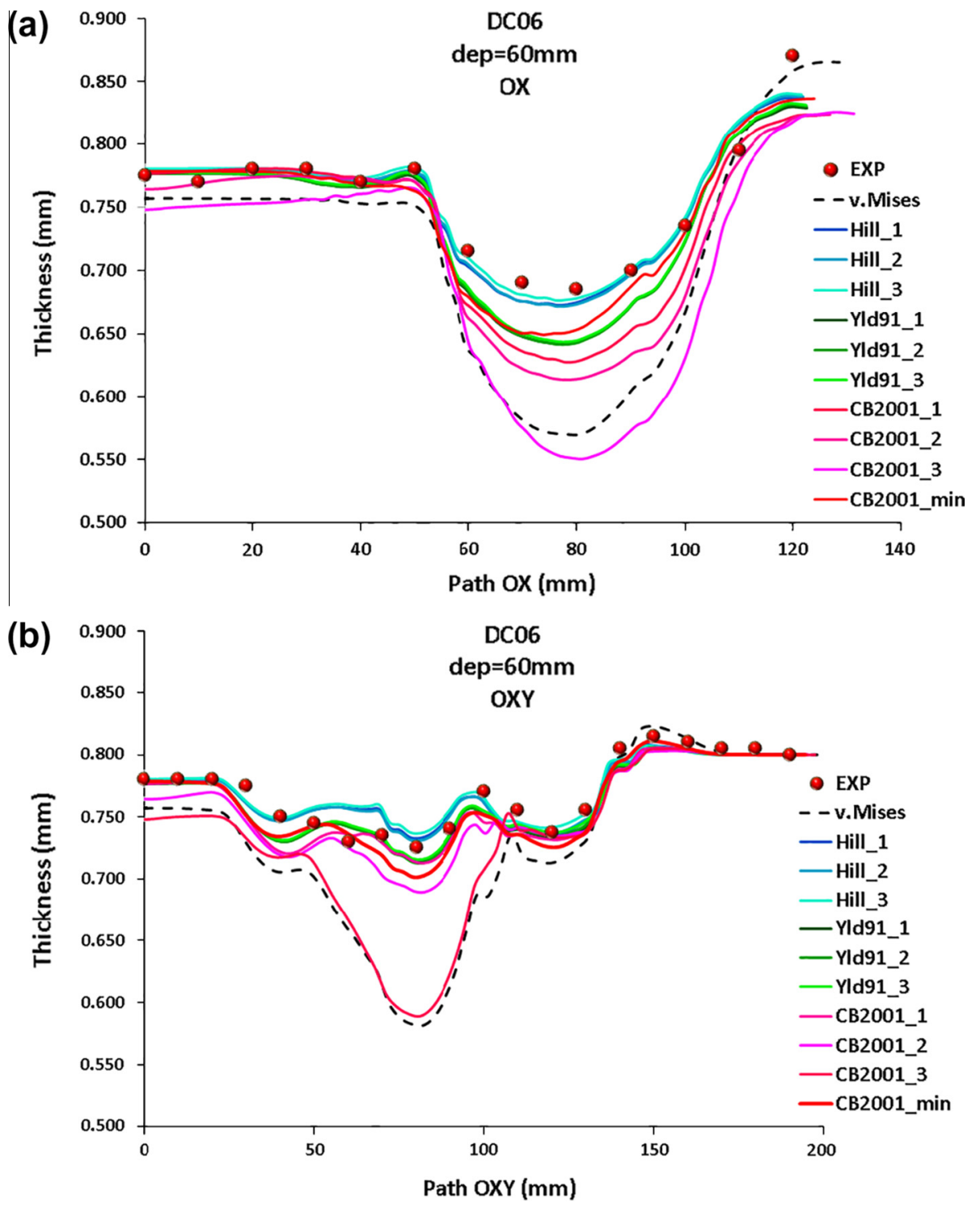

Fig. 17. Thickness evolution for DCO6 for a punch stroke of $60 \mathrm{~mm}$, along the: (a) median path OX and (b) diagonal path OXY.

discrepancy observed for DC06 steel, between predicted and experimental thickness evolutions for the different punch strokes, along the median path.

Fig. 22 shows the comparison between the flange draw-in $(s)$ as predicted for a punch stroke of $60 \mathrm{~mm}$ for the DC06 and $40 \mathrm{~mm}$ for the AA6016-T4, for all the studied yield criteria and identification strategies. The flange draw-in is defined as the distance between the current and the initial position of a material point on the border of the blank, as a function of the angle which defines its original location relative to RD. The Hill48 and Yld91 yield criteria seem to be rather insensitive to the identification strategy selected. However, flange draw-in (s) predicted by CB2001 is strongly influenced by the calibration strategy, only being asymmetric about the $45^{\circ}$ orientation to RD if the identification strategy uses experimental yield stresses and $r$-values for 7 different orientations to RD. For DC06, the flange draw-in predicted by von Mises and the CB2001_3 calibration strategy are different, although the yield surfaces in the $\sigma_{1} v s . \sigma_{2}$ plane and the thickness predictions are quite similar (see Figs. 15-17), reflecting the importance of the predicted directional stresses for predicting the material flow. For AA6016-T4, less scatter between the predicted flange draw-in $(s)$ is observed, compared to DC06, indicating the paramount role of inplane anisotropy in the draw-in profiles. The flange draw-in analysis highlights the importance of the in-plane yield stresses and $r$-values for evaluating the material flow. Therefore, in components presenting a non-symmetrical distribution of the draw-in it is more important to try to ensure adequate knowledge of the experimental directional yield stresses and $r$-values and adopt more flexible yield criteria, even if biaxial experimental values are not available. 

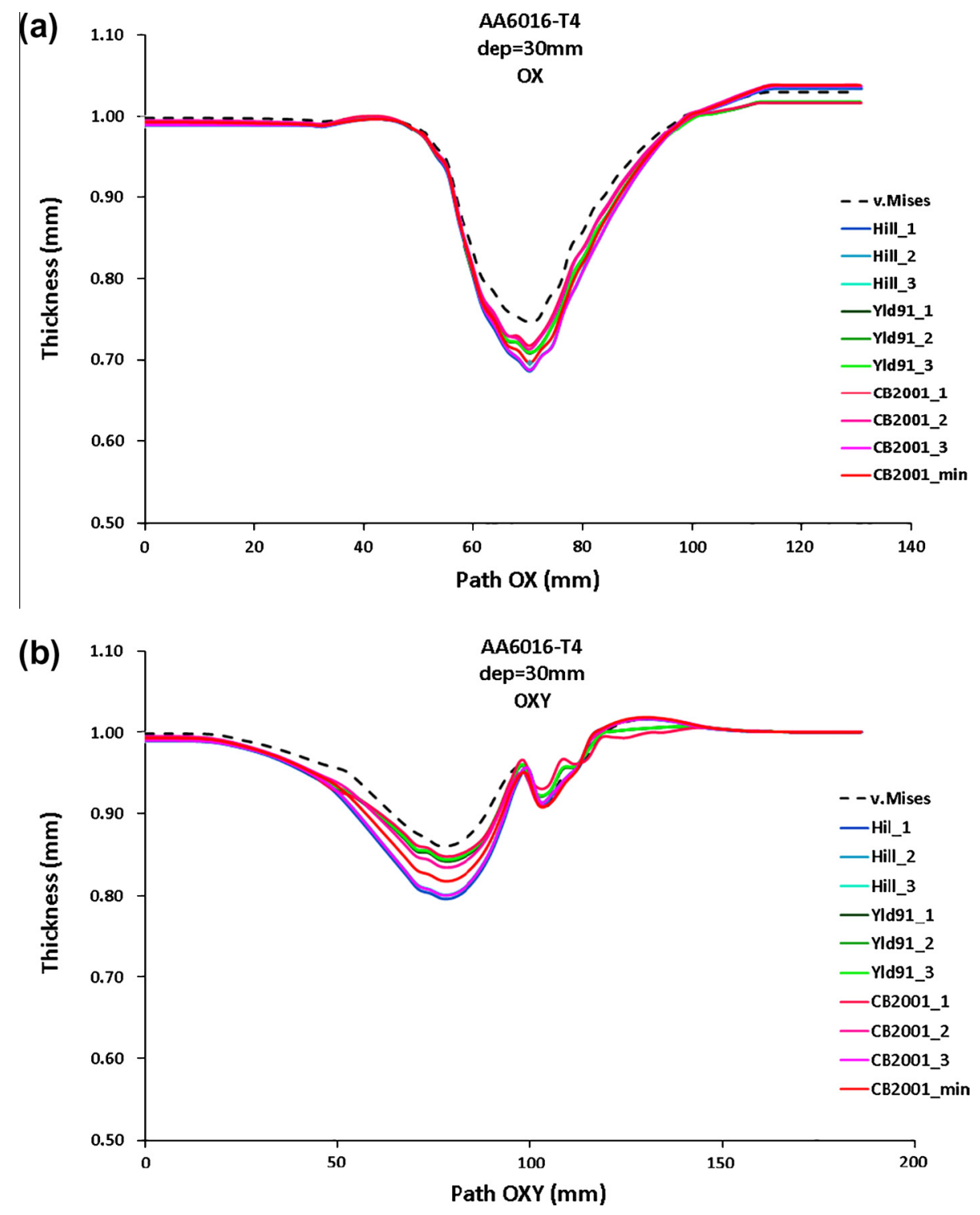

Fig. 18. Thickness evolution for AA6016-T4 for a punch stroke of $30 \mathrm{~mm}$, along the: (a) median path OX and (b) diagonal path OXY.

\section{Conclusions}

In this work, the anisotropy parameters of the Hill48, Yld91 and CB2001 yield criteria were identified using different numbers and types of experimental results. Two materials with different anisotropic behaviours were studied, namely DC06 and AA6016-T4. Globally, the results show that the number and type of experimental data has a negligible effect on the Hill48 and Yld91 anisotropy parameters. On the contrary, CB2001 calibration is quite sensitive to the experimental data available, particularly biaxial values. Therefore, to fully explore the potential of this advanced yield criterion a complete set of experimental data comprising uniaxial tensile yield stresses and $r$-values for seven directions to RD and biaxial values should be used. This enables a more accurate description of the material flow during the forming process and, consequently, of the thickness evolutions along the formed part. This is particularly important for materials presenting higher values of the planar anisotropy coefficient and relatively small anisotropy of the directional stresses.

During the deformation process, the strain state of a material point is mainly influenced by the tool's shape, which also dictates the strain path changes. However, the strain ratio is dictated by the normal to the yield surface. Therefore, when evaluating the performance of a strategy for anisotropy parameter identification, using results for sheet metal forming processes, it is important to evaluate the strain paths and their changes. The thickness prediction for material points which follow monotonous strain paths only reflects the difference in yield surface for those strain paths, while for material points 

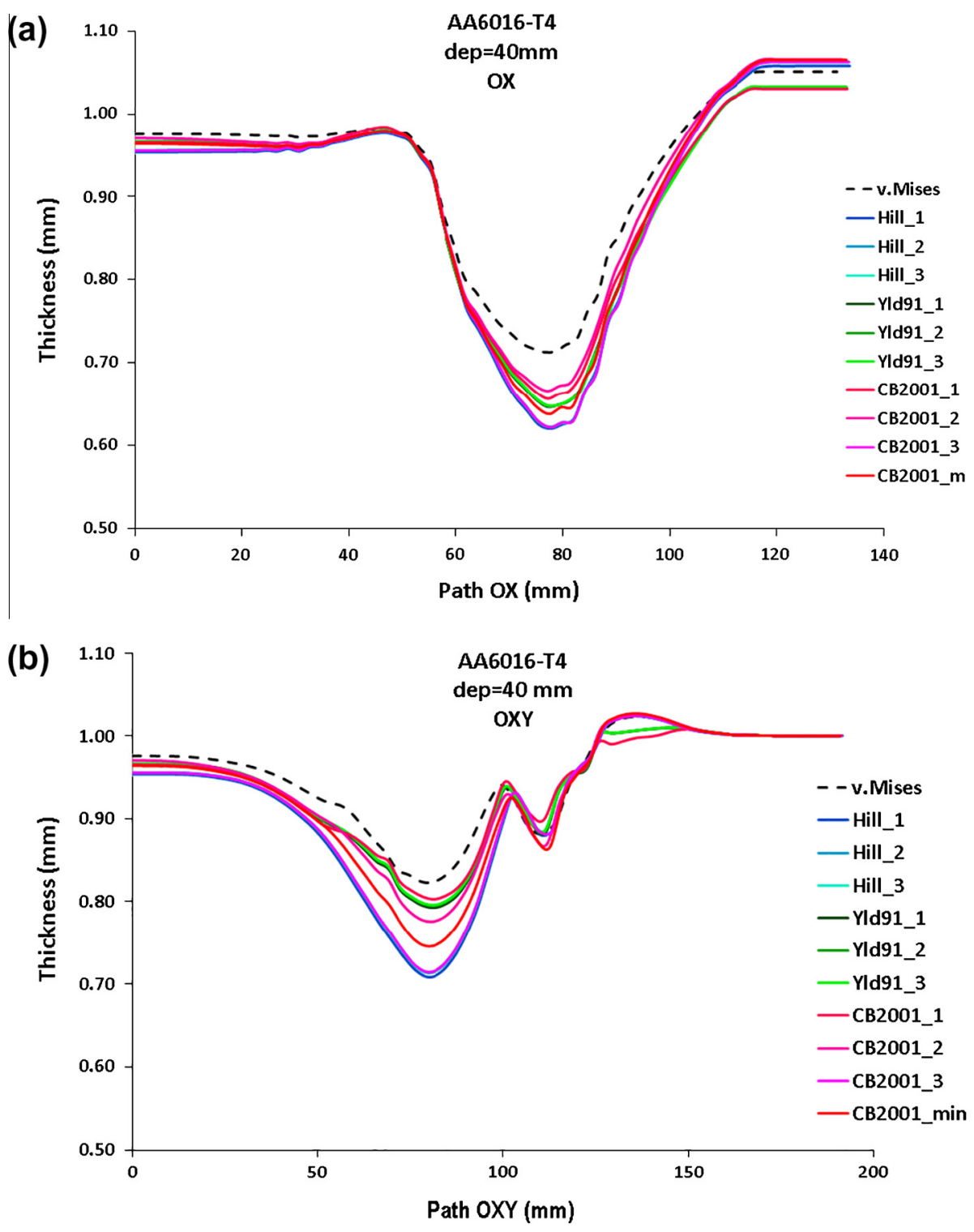

Fig. 19. Thickness evolution for AA6016-T4 for a punch stroke of $40 \mathrm{~mm}$, along the: (a) median path OX and (b) diagonal path OXY.

undergoing strain path changes it reflects the differences for a set of strain paths. Thus, the analysis of a particular instant of the forming process may not be sufficient to fully evaluate the performance of an identification strategy.

The selection of the yield criterion may not be constrained by the experimental data available. In this context, an alternative parameter identification strategy was proposed for CB2001, based on the combination of experimental results obtained from three directional tensile tests and artificial results obtained from a less flexible yield criterion (i.e. Yld91). The use of this minimum set of experimental data inhibits an improved description of directional yield stresses and $r$-values, which become similar to the ones predicted by Ydd91. Nonetheless, the results show that it is possible to use an incomplete set of experimental data to calibrate CB2001 anisotropy parameters by resorting to artificial data. In the worst case, the numerical prediction for punch force evolution, thickness distributions and material flow will be similar to the ones obtained with the Yld91. Therefore, a similar concept can be applied whenever the information concerning biaxial data is not available, keeping in mind that the success of this approach depends on the accuracy of the artificial input data determined from less flexible yield functions. Therefore, the adoption of this kind of calibration strategy is an interesting approach and it can help to increase the application of advanced models in sheet metal forming industries. 

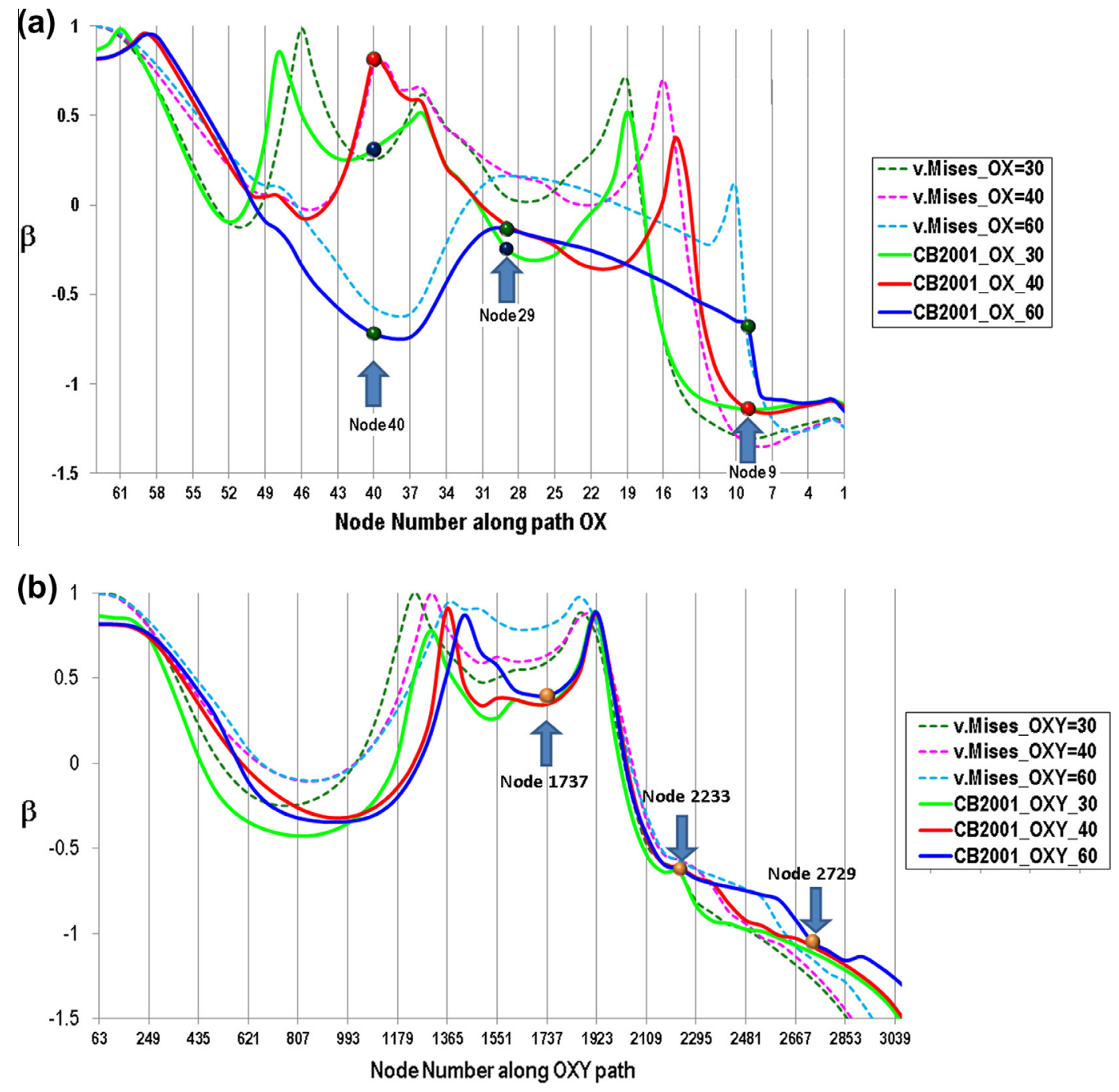

Fig. 20. Loading modes undergone by DC06 material points along the: (a) median path and (b) diagonal path.
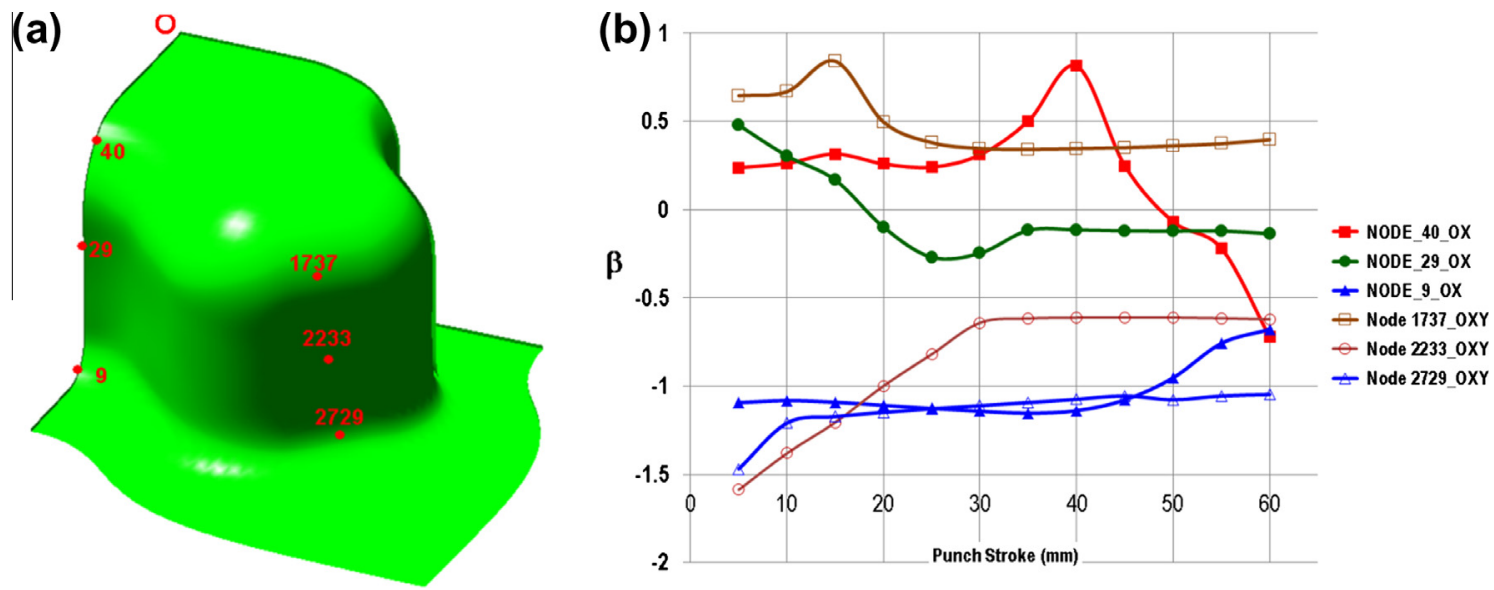

Fig. 21. (a) Location of particular material points in the formed part; (b) Strain paths undergone by those points of the cross shaped cup during punch displacement. 

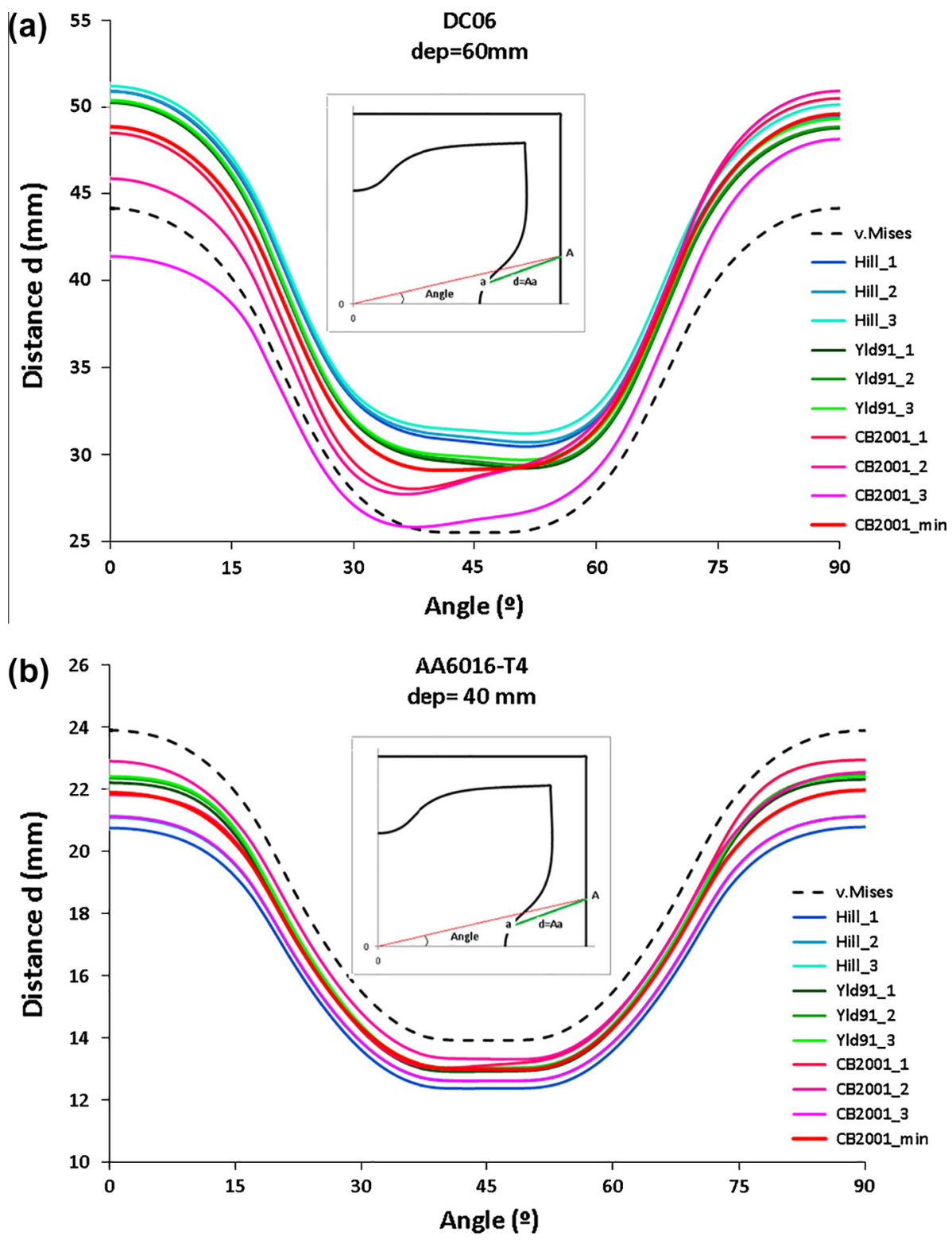

Fig. 22. Flange draw-in (s) obtained using all the identified models for a punch stroke of $40 \mathrm{~mm}$ for: (a) DC06 and (b) AA6016-T4.

\section{Acknowledgments}

The authors gratefully acknowledge the financial support of the Portuguese Foundation for Science and Technology (FCT) via the projects PTDC/EMS-TEC/1805/2012 and PEst-C/EME/UI0285/2013 and by FEDER funds through the program COMPETE - Programa Operacional Factores de Competitividade, under the project CENTRO-07-0224-FEDER-002001 (MT4MOBI). The first author is also grateful for the Post-Doc grant.

\section{References}

[1] R. Hill, A theory of the yielding flow of anisotropic metals, Proc. Roy. Soc. A193 (1948) 281-297.

[2] A.V. Hershey, The plasticity of an isotropic aggregate of anisotropic face centered cubic crystals, J. Appl. Mech. 30 (1954) 241.

[3] W.F. Hosford, A generalized isotropic yield function, J. Appl. Mech. 39 (1972) 607-619.

[4] R.W. Logan, W.F. Hosford, Upper-bound anisotropic yield locus calculations assuming (111)-pensil glide, Int. J. Mech. Sci. 22 (1980) 419-430.

[5] F. Barlat, D.J. Leg, J.C. Brem, A six-component yield function for anisotropic materials, Int. J. Plast. 7 (1991) $693-712$.

[6] A.P. Karafillis, M.C. Boyce, A general anisotropic yield criterion using bounds and a transformation weighting tensor, J. Mech. Phys. Solids 41 (1993) 1859-1886. 
[7] F. Barlat, J.W. Yoon, On linear transformations of stress tensors for the description of plastic anisotropy, Int. J. Plast. 23 (2007) 876-896.

[8] F. Barlat, Y. Maeda, K. Chung, M. Yanagawa, J.C. Brem, Y. Hayashida, D.J. Lege, K. Matsui, S.J. Murtha, S. Hattori, R.C. Becker, S. Makosey, Yield function development for aluminium alloy sheets, J. Mech. Phys. Solids 45 (1997) 1727-1763.

[9] F. Barlat, J.C. Brem, J.W. Yoon, K. Chung, R.E. Dick, D.J. Lege, F. Pourboghrat, S.-H. Choi, E. Chu, Plane stress yield function for aluminum alloy sheets part 1: theory, Int. J. Plast. 19 (2003) 1297-1319.

[10] F. Barlat, H. Aretz, J.W. Yoon, M.E. Brem, R.E. Dick, Linear transformation-based anisotropic yield functions, Int. J. Plast. (2005) $1009-1039$.

[11] L. Xu, F. Barlat, D.C. Ahn, Constitutive modeling of ferritic stainless steel, Int. J. Mat. Form. 3 (2009) 135-145.

[12] F. Barlat, J. Lian, Plastic behavior and stretchability of sheet metals. Part I: a yield function for orthotropic sheet under plane stress conditions, Int. J. Plast. 5 (1989) 55-66.

[13] H. Aretz, F. Barlat, New convex yield functions for orthotropic metal plasticity, Int. J. N-Linear Mech. 51 (2013) $97-111$.

[14] R. Hill, The Mathematical Theory of Plasticity, Oxford Clarendon Press, 1950.

[15] M. Gotoh, A theory of plastic anisotropy based on a yield function of fourth order (Plane stress state), Int. J. Mech. Sci. 19 (1977) 505-520.

[16] D.C. Drucker, Relation of experiments to mathematical theories of plasticity, J. Appl. Mech. 16 (1949) $349-357$.

[17] S. Soare, J.W. Yoon, O. Cazacu, F. Barlat, Applications of a recently proposed anisotropic yield function to sheet forming, in: Advanced Methods in Material Forming, Springer, 2007.

[18] S. Soare, J.W. Yoon, O. Cazacu, On the use of homogeneous polynomials to develop anisotropic yield functions with applications to sheet forming, Int. J. Plast. 24 (2008) 915-944.

[19] D. Banabic, T. Balan, D.S. Comsa, A New Yield Criterion for Orthotropic Sheet Metals Under Plane Stress Condition, TPR2000, Cluj-Napoca, Romania, 2000.

[20] D. Banabic, H. Arets, D.S. Comsa, L. Paraianu, An improved analytical description of orthotropy in metallic sheets, Int. J. Plast. 21 (2005) 493-512.

[21] D. Banabic, D.S. Comsa, M. Sester, Mike Selig, W. Kubli, K. Mattiasson and M. Sigvant, «Influence of constitutive equations on the accuracy of prediction in sheet metal forming simulation, in: 7th International Conference and Workshop on Numerical Simulation of 3D Sheet Metal Forming Processes, Interlaken Switzerland, 2008.

[22] H. Aretz, Applications of a new plane stress yield function to orthotropy steel and aluminum sheet metals, Modell. Simul. Mater. Sci. Eng. 12 (2004) 491-509.

[23] H. Aretz, A non quadratic plane stress yield function for orthotropic sheet metals, J. Mater. Process. Technol. 168 (2005) 1-9.

[24] D.S. Comsa, D. Banabic, Plane stress yield criterion for highly anisotropic sheet metals, in. In: 7th International Conference and Workshop on Numerical Simulation of 3D Sheet Metal Forming Processes, Interlaken Switzerland, 2008.

[25] M. Vrh, M. Halilovic, B. Starman, B. Stok, D.S. Comsa, D. Banabic, Capability of the BBC2008 yield criterion in predicting the earing profile in cup deep drawing simulations, Euro. J. Mech. A/Solids 45 (2014) 59-74.

[26] L. Lazarescu, I. Coibanu, I.P. Nicodim, D.S. Comsa, D. Banabic, Effect of the mechanical parameters used as input data in the yield criteria on the accuracy of the finite element simulation of sheet metal forming processes, Key Eng. Mater. 544-557 (2013) 204-209.

[27] K.A. Malo, O.S. Hopperstad, O.G. Lademo, Calibration of anisotropic yield criteria using uniaxial tension tests and bending test, J. Mater. Process. Technol. 80-81 (1998) 538-544.

[28] P.D. Wu, M. Jain, J. Savoie, S.R. MacEwen, P. Tugcu, K.W. Neale, Evaluation of anisotropic yield functions for aluminum sheets, Int. J. Plast. 19 (2003) $121-138$.

[29] R.F. Young, J.E. Bird, J.L. Duncan, An automated hydraulic bulge tester, J. Appl. Metalwork 2 (1981) 11-18.

[30] H. Aretz, Impact of the biaxial plastic strain ration on FLD prediction, in: 9th Esaform Conference on Material Forming, Glasgow UK, 2006.

[31] L. Paraianu, D.S. Comsa, I. Nicodim, I. Ciobanu, D. Banabic, Effect of the constitutive law on the accuracy of prediction of the forming limit curves, Key Eng. Mater. 505 (2012) 77-82.

[32] H. Aretz, O.S. Hopperstad, O.G. Lademo, Yield function calibration for orthotropic sheet metals based on uniaxial and plane strain tensile tests, J. Mater. Process. Technol. 186 (2007) 221-235.

[33] O. Cazacu, F. Barlat, Generalization of Drucker's yield criterion to orthotropy, Maths. Mech. Solids 6 (2001) $613-630$.

[34] P.D. Barros, M.C. Oliveira, J.L. Alves, L.F. Menezes, Earing prediction in drawing and ironing processes using advanced yield criterion, Key Eng. Mater. 554-557 (2013) 2266-2276.

[35] H.W. Swift, Plastic instability under plane stress, J. Mech. Phys. Solids 1 (1952) 1-18.

[36] E. Voce, The relationship between stress and strain for homogeneous deformation, J. Inst. Metals 74 (1948) 537.

[37] Selection and identification of elastoplastic models for the materials used in the benchmarks, Digital Die Design Systems (3DS) Report, 2001.

[38] S. Bouvier, J.L. Alves, M.C. Oliveira, L.F. Menezes, Modelling of anisotropic work-hardening behaviour of metallic materials subjected to strain-path changes, Comput. Mater. Sci. 32 (2005) 301-315.

[39] O. Cazacu, B. Plunkett, F. Barlat, Orthotropic yield criterion for hexagonal close packed metals, Int. J. Plast. 22 (2006) $1171-1194$.

[40] F. Grytten, B. Holmedal, O.S. Hopperstad, T. Borvik, Evaluation of identification methods for YLD2004-18p, Int. J. Plast. 24 (2008) $2248-2277$.

[41] D. Banabic, T. Balan, D.-S. Comsa, W. Müller, K. Pöhlandt, Experimental validation of a new anisotropic yield criterion, in: 3rd Esaform Conference on Material Forming, Stuttgart, Germany, 2000.

[42] B.M. Chaparro, J.L. Alves, L.F. Menezes, J.V. Fernandes, Optimization of the phenomenological constitutive models parameters using genetic algorithms, in: Advanced Methods in Material Forming, Springer, 2007.

[43] S. Zhang, L. Leotoing, D. Guines, S. Thuillier, S. Zang, Calibration of anisotropic yield criterion with conventional tests or biaxial test, Int. J. Mech. Sci. 85 (2014) 142-151.

[44] J.L. Alves, M.C. Oliveira, L.F. Menezes, S. Bouvier, Influence of the yield criteria on the numerical results: the cross tool example, in: Esaform'06 Conference on Material Forming, Glasgow, UK, 2006.

[45] S. Bouvier, J.L. Alves, L.F. Menezes, M.C. Oliveira, Characterizing the plastic anisotropy and its effect on sheet metal forming simulation, in: COMPLAS VIII, Barcelona, Spain, 2005.

[46] Cashform, An Accurate Plasticity Model of Texture, Strain-path Induced Anisotropy and Forming Limits for Computer Aided Sheet Metal Forming, Technical Report, 2001.

[47] L.F. Menezes, C. Teodosiu, Three-dimensional numerical simulation of deep-drawing process using solid finite elements, J. Mate. Process. Technol. 97 (2000) 100-106. 